\title{
NEON AND CNO ABUNDANCES FOR EXTREME HELIUM STARS—A NON-LTE ANALYSIS
}

\author{
Gajendra Pandey ${ }^{1}$ and David L. LamberT ${ }^{2}$ \\ ${ }^{1}$ Indian Institute of Astrophysics, Bangalore, 560034, India; pandey@iiap.res.in \\ 2 The W.J. McDonald Observatory, University of Texas at Austin, Austin, TX 78712-1083, USA; dll@astro.as.utexas.edu \\ Received 2010 October 19; accepted 2010 November 22; published 2011 January 12
}

\begin{abstract}
A non-LTE (NLTE) abundance analysis was carried out for three extreme helium stars (EHes): BD+10 2179 , BD $-9^{\circ} 4395$, and LS IV $+6^{\circ} 002$, from their optical spectra with NLTE model atmospheres. NLTE TLUSTY model atmospheres were computed with $\mathrm{H}, \mathrm{He}, \mathrm{C}, \mathrm{N}, \mathrm{O}$, and Ne treated in NLTE. Model atmosphere parameters were chosen from consideration of fits to observed $\mathrm{He}$ I line profiles and ionization equilibria of $\mathrm{C}$ and $\mathrm{N}$ ions. The program SYNSPEC was then used to determine the NLTE abundances for $\mathrm{Ne}$ as well as $\mathrm{H}, \mathrm{He}, \mathrm{C}, \mathrm{N}$, and O. LTE neon abundances from Ne I lines in the EHes: LSE 78, V1920 Cyg, HD 124448, and PV Tel, are derived from published models and an estimate of the NLTE correction applied to obtain the NLTE Ne abundance. We show that the derived abundances of these key elements, including $\mathrm{Ne}$, are well matched with semi-quantitative predictions for the EHe resulting from a cold merger (i.e., no nucleosynthesis during the merger) of an He white dwarf with a $\mathrm{C}-\mathrm{O}$ white dwarf.
\end{abstract}

Key words: stars: abundances - stars: atmospheres - stars: chemically peculiar - stars: evolution - stars: fundamental parameters

\section{INTRODUCTION}

The principal class of hydrogen-deficient supergiant stars comprises three subclasses which in order of increasing but overlapping temperature intervals from coolest to hottest are the $\mathrm{H}$-deficient carbon stars $(\mathrm{HdC})$, the $\mathrm{R}$ Coronae Borealis stars (RCB), and the extreme helium stars (EHe). A common supposition is that the three subclasses are related in terms of origin and evolution. The origin of these very rare stars has long been disputed but it now seems likely that the majority are formed through a merger of an He white dwarf (WD) with a $\mathrm{C}-\mathrm{O}$ WD, the so-called double-degenerate (DD) scenario (Webbink 1984; Iben \& Tutukov 1984; Saio \& Jeffery 2002). Others may be the result of a final He-shell flash in a post-asymptotic giant branch (post-AGB) star, the so-called final flash (FF) scenario (Iben et al. 1983, 1996; Herwig 2001; Blöcker 2001).

Much of the evidence for deciding whether HdC, RCB, or EHe stars come from the DD or FF scenario (or neither) depends on comparison of the observed chemical composition with predictions by the two scenarios. It is in this context that we present in this paper a non-LTE (NLTE) analysis of the neon abundance of a sample of EHe stars where $\mathrm{Ne}$ I lines are prominent in optical spectra; neon is detectable in EHe stars, the warmer RCBs but not the HdCs. (The NLTE analyses are extended here to $\mathrm{He}, \mathrm{C}, \mathrm{N}$, and $\mathrm{O}$ lines.)

If reliable $\mathrm{Ne}$ abundances can be provided for EHes and RCBs, neon will join other abundances as clues to the origins and evolution of the $\mathrm{H}$-deficient supergiants. In addition to the obvious importance of $\mathrm{C}, \mathrm{N}$, and $\mathrm{O}$ elemental abundances, one may now note a variety of other abundance anomalies peculiar to these supergiants including, for example, the presence of lithium in a subset of RCBs and one HdC (Asplund et al. 2000; Rao \& Lambert 1996), the large overabundance of fluorine in EHes and RCBs (Pandey 2006; Pandey et al. 2008), high concentrations of ${ }^{18} \mathrm{O}$ (relative to ${ }^{16} \mathrm{O}$ ) in HdCs and cool RCBs (Clayton et al. 2005, 2007; García-Hernández et al. 2009, 2010), extraordinary high $\mathrm{Si} / \mathrm{Fe}$ and $\mathrm{S} / \mathrm{Fe}$ ratios in the "minority" RCBs (Rao \& Lambert 1996).
If neon is to provide an effective addition to the list of abundance anomalies, its abundance must be determined reliably and, in this regard, the primary consideration would appear to be an adequate treatment of NLTE effects in the formation of the observable neon lines. Realization that NLTE effects are considerable for optical Ne I lines arose from pioneering calculations by Auer \& Mihalas (1973) for normal B-type stars with effective temperatures of around 20,000 K. These authors showed that the Ne abundance derived by accounting for NLTE effects was about a factor of five less than that given by LTE. Not only was this the first result showing major NLTE effects on abundances for hot stars but the NLTE Ne abundance was shown to be in good agreement with that for H II regions as derived from emission lines. The origin of the marked NLTE effects is discussed by Auer \& Mihalas. A key ingredient is that the ultraviolet $\mathrm{Ne}$ I resonance lines are optically thick-see a concise discussion by Cunha et al. (2006) who report on modern calculations of Ne NLTE effects as applied to B stars in the Orion Association. Given that the ultraviolet resonance lines may be similarly optically thick in atmospheres of EHe stars, it became apparent that addition of neon to the list of referees between DD and FF scenarios would require evaluation of the NLTE effects on the observable neon lines.

In the following sections, we successively describe our optical spectra, the NLTE calculations including a sanity check involving our analysis of normal B stars previously discussed by Cunha et al. (2006) and Morel \& Butler (2008), our abundance analyses of seven EHes, a discussion of the DD scenario with a comparison of semi-quantitative predictions with the observationally based abundances of $\mathrm{He}, \mathrm{C}, \mathrm{O}$, and $\mathrm{Ne}$ as well as remarks on abundances not determinable for EHes (e.g., Li, ${ }^{18} \mathrm{O}$, and $\mathrm{F}$ ). This comparison is followed by remarks on the FF scenario and a few concluding remarks.

\section{OBSERVATIONS}

High-resolution optical spectra of $\mathrm{BD}+10^{\circ} 2179, \mathrm{BD}-9^{\circ}$ 4395, and V1920 Cyg were obtained on 1998 January 24, 2000 June 16, and 1996 July 25 , respectively, at the coudé focus of the 


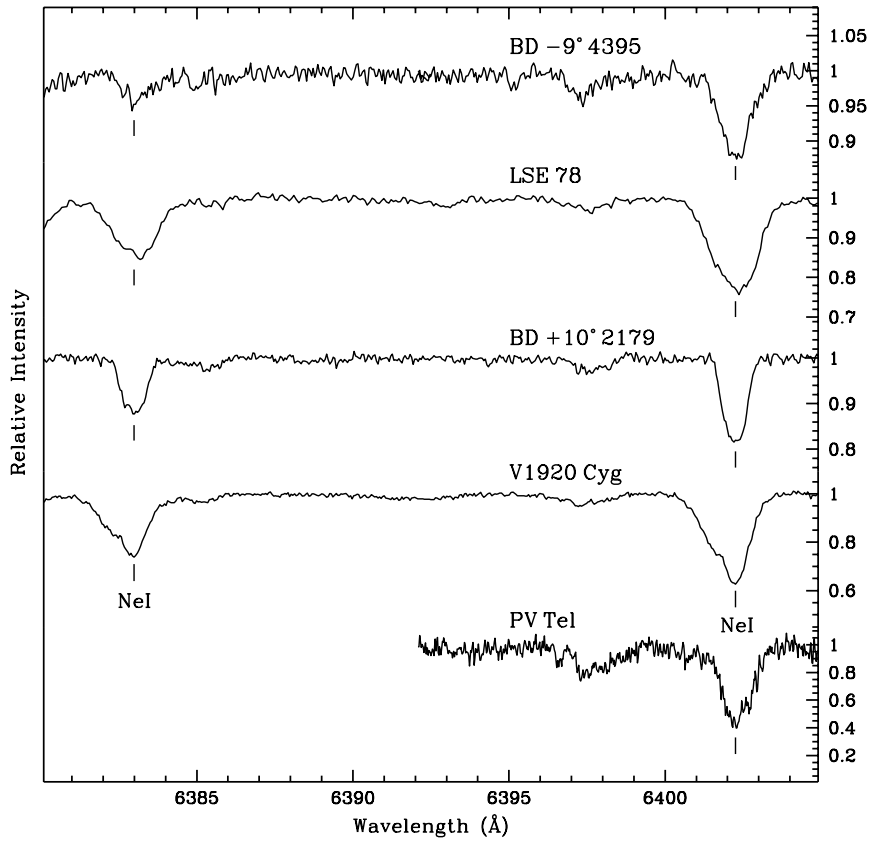

Figure 1. Spectral region from 6380 to $6405 \AA$ is shown for five EHes with the hottest star at the top and the coolest star at the bottom. The Ne I lines at $6382.99 \AA$ and at $6402.25 \AA$ are marked.

W.J. McDonald Observatory's Harlan J. Smith 2.7-m telescope with the Robert G. Tull cross-dispersed echelle spectrograph (Tull et al. 1995) at a resolving power of $R=60,000$ except for BD-9 4395's spectrum acquired at $R=40,000$. These spectra with $R=60,000$ were previously described by Pandey et al. (2006). Additional spectra of BD-9 4395 were obtained on 2002 July 18, 22, and 25 at the W. J. McDonald Observatory's Otto Struve 2.1-m telescope with the Sandiford Caasegrain echelle spectrograph (McCarthy et al. 1993) at a resolving power of $R=40,000$. The spectrum of LSE 78 was acquired with the Cassegrain echelle spectrograph at CTIO, and the observations are described in Pandey \& Reddy (2006). Finally, spectra at $R=$ 30,000 of the two southern EHes-PV Tel and HD 124448 - are from the Vainu Bappu Observatory and the fiber-fed crossdispersed echelle spectrometer (Rao et al. 2004, 2005) at the 2.34-m telescope. The Image Reduction and Analysis Facility (IRAF) software package was used to reduce all spectra. ${ }^{3}$

Sample wavelength intervals in Figures 1 and 2 include one or two of the Ne I lines with the EHes ordered from top to bottom in order of decreasing effective temperature. All spectra are aligned to the rest wavelengths of well-known lines. Inspection of the figures shows that the line profiles are not always symmetric. Asymmetries obviously present in the case of LSE 78 and V1920 Cyg are most probably due to atmospheric pulsations. In the case of V1920 Cyg, another observation on 1996 July 26, one day following the spectrum illustrated in Figures 1 and 2, showed symmetric line profiles with no appreciable change in the equivalent widths of lines. The equivalent width change translates to an abundance change of less than 0.1 dex. In the case of $\mathrm{BD}-9^{\circ} 4395$, emission components may appear and disappear. Variable photospheric spectra for EHes are common (Jeffery 2008) with V1920 Cyg representative of the variability and BD-9 4395 as an extreme example. Nonetheless, we assume that models constructed with classical assumptions

\footnotetext{
3 The IRAF software is distributed by the National Optical Astronomy Observatories under contract with the National Science Foundation.
}

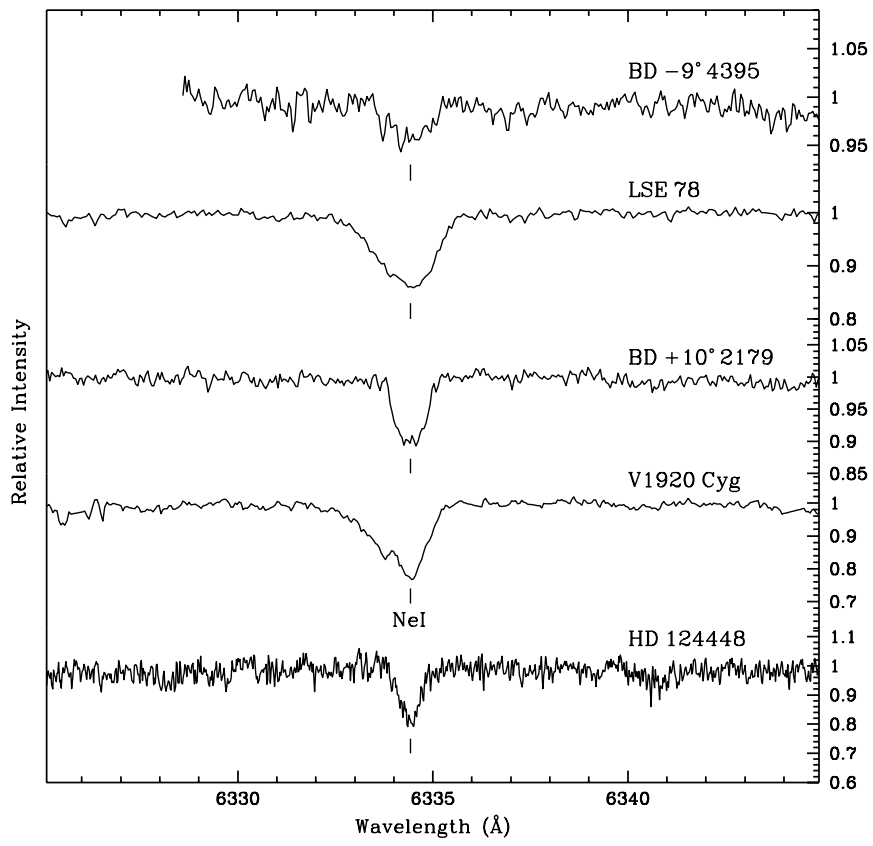

Figure 2. Spectral region from 6325 to $6345 \AA$ is shown for five EHes with the hottest star at the top and the coolest star at the bottom. The Ne I line at $6334.43 \AA$ is marked.

(plane parallel layers in hydrostatic equilibrium) are adequate for our purpose.

\section{NLTE ATMOSPHERES AND ABUNDANCES}

Our calculations use codes developed by Hubeny and colleagues: the program TLUSTY for calculating LTE and NLTE model atmospheres (Hubeny 1988; Hubeny \& Lanz 1995) and the spectrum synthesis code SYNSPEC (Hubeny et al. 1994). In exercising these codes, we adopt the atomic data and model atoms provided on the TLUSTY homepage ${ }^{4}$ (Lanz \& Hubeny 2007, 2003).

The suite of codes was imported to Bangalore and run by one of us (GP) on a local computer. Before proceeding to construct and apply $\mathrm{H}$-deficient model atmospheres, our imported codes were tested for normal B-type stars. In particular, we computed an NLTE model atmosphere for HD 35299, a normal B star in the Orion sample for which Cunha et al. (2006) derived NLTE Ne abundances. The NLTE TLUSTY model was computed for the stellar parameters adopted by Cunha et al.: $T_{\text {eff }}=24,000 \mathrm{~K}, \log g=4.25 \mathrm{cgs}$, and a microturbulence of $2 \mathrm{~km} \mathrm{~s}^{-1}$ and solar abundances. The $g f$-values for the Ne I lines are taken from Seaton (1998) who showed that Opacity Project theoretical $g f$-values are in very good agreement not only with theoretical calculations of comparable sophistication but also with experimental determinations. Then, the NLTE Ne abundances were computed using the TLUSTY model by matching the observed equivalent width of $\mathrm{Ne}$ I lines with NLTE predictions from running the SYNSPEC code. Observed equivalent widths were kindly provided by Dr. Katia Cunha (2010, private communication) for the eight lines measured by them. Our NLTE Ne abundance for the eight lines is $\log (\mathrm{Ne})=$ $8.20 \pm 0.08$ in good agreement with the value of 8.18 given by Cunha et al. (2006). This agreement over NLTE Ne abundances is taken as evidence that our implementation of the TLUSTYrelated codes was successful.

\footnotetext{
4 http://nova.astro.umd.edu/index.html
} 
As a second check, we analyzed $\mathrm{Ne}$ I and $\mathrm{Ne}$ II lines in $\beta \mathrm{CMa}$. Morel \& Butler (2008) analyzed seven Ne I and four Ne II lines in this star. Morel \& Butler compute the NLTE Ne abundances for an LTE Kurucz model with the parameters $T_{\text {eff }}=24,000 \mathrm{~K}$, $\log g=3.5 \mathrm{cgs}$ and a microturbulence of $14 \mathrm{~km} \mathrm{~s}^{-1}$. We computed an NLTE TLUSTY model for these stellar parameters. The $g f$-values and the measured equivalent widths of the $\mathrm{Ne}$ I and the Ne II lines were taken from Morel \& Butler. NLTE Ne abundances were computed using the TLUSTY atmosphere and model atoms by matching the measured width of $\mathrm{Ne}$ I and Ne II lines with NLTE predictions from the SYNSPEC code. Our NLTE Ne abundance for the seven $\mathrm{Ne}$ I lines is $\log (\mathrm{Ne})=$ $7.89 \pm 0.09$ in agreement with the value of $7.89 \pm 0.04$ given by Morel \& Butler. For the three Ne II lines, our NLTE Ne abundance is $\log (\mathrm{Ne})=8.16 \pm 0.16$, where the value of $7.89 \pm$ 0.06 is given by Morel \& Butler. Note that one of the four $\mathrm{Ne}$ II lines returns a higher abundance and was not included in calculating our mean abundance. The different Ne abundances from $\mathrm{Ne}$ II lines are noted and may arise from the use of different models (TLUSTY NLTE versus Kurucz LTE) and the use of different model Ne atoms. These checks on published NLTE Ne abundances are taken as evidence that our implementation of the TLUSTY-related codes was successful.

A small grid of NLTE TLUSTY model atmospheres for EHe stars was computed for $T_{\text {eff }}$ from $15,000 \mathrm{~K}$ to $31,000 \mathrm{~K}$ and surface gravities $\log g=2.35$ to 4.3 . The abundances adopted for the grid were representative of the LTE abundances given by Pandey et al. (2006). In particular, the $\mathrm{C} / \mathrm{He}$ ratio was assumed to be $1 \%$. Sample models for $\mathrm{H} / \mathrm{He}=0.1$ and 0.0001 showed that the derived abundances of neon and other elements are insensitive to the $\mathrm{H}$ abundance in this range.

\section{NLTE ABUNDANCE ANALYSES}

\section{1. $B D+10^{\circ} 2179$}

An extensive LTE abundance analysis of BD+10 2179 was reported by Pandey et al. (2006) from optical and ultraviolet spectra. Abundances were obtained for 18 elements from $\mathrm{H}$ to $\mathrm{Zn}$ but neon was not included. Here, we present an NLTE model atmosphere redetermination of the $\mathrm{He}, \mathrm{C}, \mathrm{N}$, and $\mathrm{O}$ abundances and the first determination of the Ne abundance. The star's atmospheric parameters are reassessed using NLTE atmospheres and NLTE line formation for $\mathrm{He}, \mathrm{C}, \mathrm{N}, \mathrm{O}$, and $\mathrm{Ne}$ lines.

Optical lines of He I, C I-III, N II, O II, and $\mathrm{Ne}_{\mathrm{I}}$ are used. Details about these lines except for $\mathrm{Ne}$ I are taken from Table 2 of Pandey et al. (2006). Details include a line's $g f$-value and the reference to the source of that value, its lower excitation potential $(\chi)$, and information on the line's Stark and radiative damping constants. Values from 2006 are adopted here in full. For Ne, which is not in the 2006 table, we adopt the $g f$-values from Seaton (1998), as noted above. Table 1 of this paper lists the chosen lines of $\mathrm{C}, \mathrm{N}, \mathrm{O}$, and $\mathrm{Ne}$ where the equivalent widths of $\mathrm{Ne}$ I lines were measured off the spectrum used for the 2006 analysis.

Atmospheric parameters are obtained by the procedures used for the 2006 LTE analysis but using NLTE TLUSTY model atmospheres and NLTE line formation using the TLUSTY model atoms. The microturbulence is provided from the usual requirement that the abundance from lines of a given species be independent of a line's equivalent width: we use the C II lines. The effective temperature and surface gravity are found from intersecting loci in the $\left(T_{\text {eff }}, \log g\right)$ plane with loci provided by
Table 1

Photospheric Line by Line NLTE and LTE Abundances, and the Line's Measured Equivalent Width $\left(W_{\lambda}\right)$ in $\mathrm{m} \AA$ for $\mathrm{BD}+10^{\circ} 2179$

\begin{tabular}{|c|c|c|c|c|c|}
\hline \multirow[t]{2}{*}{ Line } & \multirow{2}{*}{$\begin{array}{c}\chi \\
(\mathrm{eV})\end{array}$} & \multirow[t]{2}{*}{$\log g f$} & \multirow{2}{*}{$\begin{array}{c}W_{\lambda} \\
(\mathrm{m} \AA)\end{array}$} & \multicolumn{2}{|c|}{$\log \epsilon(X)$} \\
\hline & & & & NLTE $^{\mathrm{a}}$ & $\mathrm{LTE}^{\mathrm{b}}$ \\
\hline $\mathrm{H}_{\mathrm{I}} \lambda 3970.072$ & 10.199 & -0.993 & 90 & 8.26 & 8.60 \\
\hline Н г $\lambda 4101.734$ & 10.199 & -0.753 & 146 & 8.28 & 8.60 \\
\hline Н г $\lambda 4340.462$ & 10.199 & -0.447 & 242 & 8.32 & 8.64 \\
\hline Н г $\lambda 4861.323$ & 10.199 & -0.020 & 430 & 8.54 & 8.88 \\
\hline Mean... & $\ldots$ & $\ldots$ & $\ldots$ & $8.35 \pm 0.13$ & $8.68 \pm 0.13$ \\
\hline C I $\lambda 4932.049$ & 7.685 & -1.658 & 13 & 9.33 & 9.41 \\
\hline $\mathrm{C}_{\mathrm{I}} \lambda 5052.167$ & 7.685 & -1.303 & 28 & 9.37 & 9.42 \\
\hline Mean. .. & $\ldots$ & $\ldots$ & $\ldots$ & $9.35 \pm 0.03$ & $9.42 \pm 0.01$ \\
\hline $\mathrm{C}_{\text {II }} \lambda 3918.980$ & 16.333 & -0.533 & 286 & 9.08 & 9.30 \\
\hline C II $\lambda 3920.690$ & 16.334 & -0.232 & 328 & 9.01 & 9.28 \\
\hline C II $\lambda 4017.272$ & 22.899 & -1.031 & 43 & 9.40 & 9.40 \\
\hline $\mathrm{C}_{\text {II }} \lambda 4021.166$ & 22.899 & -1.333 & 27 & 9.40 & 9.40 \\
\hline C II $\lambda 4306.330$ & 21.150 & -1.684 & 46 & 9.25 & 9.32 \\
\hline $\mathrm{C}_{\text {II }} \lambda 4307.581$ & 20.150 & -1.383 & 77 & 9.33 & 9.42 \\
\hline $\mathrm{C}_{\text {II }} \lambda 4313.100$ & 23.120 & -0.373 & 83 & 9.40 & 9.38 \\
\hline C II $\lambda 4317.260$ & 23.120 & -0.005 & 113 & 9.35 & 9.36 \\
\hline $\mathrm{C}_{\text {II }} \lambda 4318.600$ & 23.120 & -0.407 & 80 & 9.40 & 9.38 \\
\hline C II $\lambda 4321.650$ & 23.120 & -0.901 & 45 & 9.43 & 9.41 \\
\hline $\mathrm{C}_{\text {II }} \lambda 4323.100$ & 23.120 & -1.105 & 45 & 9.64 & 9.62 \\
\hline C II $\lambda 4637.630$ & 21.150 & -1.229 & 75 & 9.21 & 9.27 \\
\hline C II $\lambda 4867.066$ & 19.495 & -1.781 & 35 & 9.27 & 9.10 \\
\hline $\mathrm{C}_{\text {II }} \lambda 5032.128$ & 20.922 & -0.143 & 174 & 9.40 & 9.45 \\
\hline C II $\lambda 5035.943$ & 20.920 & -0.399 & 113 & 9.09 & 9.14 \\
\hline $\mathrm{C}_{\text {II }} \lambda 5125.208$ & 20.150 & -1.597 & 51 & 9.36 & 9.40 \\
\hline C II $\lambda 5126.963$ & 20.150 & -1.899 & 32 & 9.36 & 9.40 \\
\hline $\mathrm{C}_{\text {II }} \lambda 5137.257$ & 20.701 & -0.911 & 91 & 9.32 & 9.36 \\
\hline C II $\lambda 5139.174$ & 20.704 & -0.707 & 118 & 9.38 & 9.43 \\
\hline $\mathrm{C}_{\text {II }} \lambda 5143.495$ & 20.704 & -0.212 & 166 & 9.31 & 9.38 \\
\hline C II $\lambda 5145.165$ & 20.710 & +0.189 & 207 & 9.34 & 9.31 \\
\hline C II $\lambda 5151.085$ & 20.710 & -0.179 & 169 & 9.37 & 9.37 \\
\hline Mean. .. & $\ldots$ & $\ldots$ & $\ldots$ & $9.32 \pm 0.14$ & $9.36 \pm 0.11$ \\
\hline C III $\lambda 4186.900$ & 40.010 & +0.918 & 19 & 9.29 & 9.99 \\
\hline $\mathrm{C}_{\text {III }} \lambda 4647.420$ & 29.535 & +0.070 & 51 & $10.01 ?$ & 9.39 \\
\hline C III $\lambda 4650.250$ & 29.535 & -0.151 & 42 & $10.05 ?$ & 9.40 \\
\hline C III $\lambda 4651.470$ & 29.535 & -0.629 & 27 & $10.15 ?$ & 9.43 \\
\hline Mean... & $\ldots$ & $\ldots$ & $\ldots$ & $9.29 \pm 0.00$ & $9.41 \pm 0.02$ \\
\hline N II $\lambda 3842.180$ & 21.150 & -0.692 & 30 & 8.21 & 8.13 \\
\hline N II $\lambda 3955.851$ & 18.466 & -0.813 & 68 & 8.14 & 8.15 \\
\hline N II $\lambda 3994.996$ & 18.498 & +0.208 & 139 & 7.87 & 7.95 \\
\hline N II $\lambda 4179.670$ & 23.250 & -0.204 & 23 & 8.29 & 8.12 \\
\hline N II $\lambda 4227.740$ & 21.600 & -0.061 & 42 & 8.07 & 7.95 \\
\hline $\mathrm{N}_{\text {II }} \lambda 4447.030$ & 20.411 & +0.228 & 75 & 7.94 & 7.87 \\
\hline N II $\lambda 4507.560$ & 20.666 & -0.817 & 25 & 8.24 & 8.12 \\
\hline N II $\lambda 4601.480$ & 18.468 & -0.428 & 79 & 8.00 & 8.00 \\
\hline N II $\lambda 4607.160$ & 18.464 & -0.507 & 73 & 8.01 & 8.01 \\
\hline N II $\lambda 4613.870$ & 18.468 & -0.665 & 61 & 8.02 & 8.00 \\
\hline N II $\lambda 4643.090$ & 18.484 & -0.359 & 88 & 8.05 & 8.06 \\
\hline N II $\lambda 4654.531$ & 18.497 & -1.404 & 20 & 8.04 & 7.99 \\
\hline N II $\lambda 4667.208$ & 18.497 & -1.533 & 20 & 8.17 & 8.12 \\
\hline N II $\lambda 4674.908$ & 18.497 & -1.463 & 19 & 8.07 & 8.02 \\
\hline N II $\lambda 4779.720$ & 20.650 & -0.587 & 34 & 8.27 & 8.14 \\
\hline N II $\lambda 4781.190$ & 20.650 & -1.308 & 9 & 8.22 & 8.10 \\
\hline N II $\lambda 4788.130$ & 20.650 & -0.363 & 35 & 8.07 & 7.94 \\
\hline $\mathrm{N}_{\text {II }} \lambda 4810.310$ & 20.660 & -1.084 & 17 & 8.35 & 8.21 \\
\hline N II $\lambda 4895.117$ & 17.877 & -1.338 & 18 & 7.76 & 7.72 \\
\hline $\mathrm{N}_{\text {II }} \lambda 5002.700$ & 18.480 & -1.022 & 42 & 8.16 & 8.09 \\
\hline $\mathrm{N}_{\text {II }} \lambda 5007.328$ & 20.940 & +0.171 & 52 & 7.98 & 7.84 \\
\hline N II $\lambda 5010.620$ & 18.470 & -0.607 & 71 & 8.12 & 8.08 \\
\hline N II $\lambda 5025.659$ & 20.666 & -0.547 & 28 & 8.17 & 8.03 \\
\hline N II $\lambda 5045.090$ & 18.460 & -0.407 & 87 & 8.10 & 8.07 \\
\hline Mean... & $\ldots$ & $\ldots$ & $\ldots$ & $8.10 \pm 0.14$ & $8.03 \pm 0.11$ \\
\hline O II $\lambda 4072.157$ & 25.643 & +0.552 & 26 & 7.92 & 7.60 \\
\hline O II $\lambda 4185.449$ & 28.351 & +0.604 & 11 & 8.14 & 7.69 \\
\hline O II $\lambda 4189.789$ & 28.354 & +0.717 & 20 & 8.47 & 7.99 \\
\hline
\end{tabular}


Table 1

(Continued)

\begin{tabular}{cccccc}
\hline \hline Line & $\chi$ & $\log g f$ & $W_{\lambda}$ & \multicolumn{2}{c}{$\log \epsilon(\mathrm{X})$} \\
\cline { 5 - 6 } & $(\mathrm{eV})$ & & $(\mathrm{m} \AA)$ & NLTE $^{\mathrm{a}}$ & LTE $^{\mathrm{b}}$ \\
\hline O II $\lambda 4336.860$ & 22.973 & -0.763 & 11 & 7.93 & 7.69 \\
O II $\lambda 4345.567$ & 22.979 & -0.346 & 18 & 7.81 & 7.59 \\
O II $\lambda 4349.426$ & 22.993 & +0.060 & 32 & 7.79 & 7.61 \\
O II $\lambda 4366.888$ & 22.993 & -0.348 & 15 & 7.71 & 7.49 \\
O II $\lambda 4414.901$ & 23.435 & +0.172 & 33 & 7.88 & 7.66 \\
OII $\lambda 4416.973$ & 23.413 & -0.077 & 26 & 7.97 & 7.72 \\
O II $\lambda 4641.817$ & 22.973 & +0.055 & 32 & 7.87 & 7.69 \\
O II $\lambda 4649.143$ & 22.993 & +0.308 & 41 & 7.79 & 7.65 \\
Mean. & $\ldots$ & $\ldots$ & $\ldots$ & $7.93 \pm 0.21$ & $7.67 \pm 0.12$ \\
Ne I $\lambda 5852.488$ & 16.850 & -0.490 & 61 & 7.78 & 8.54 \\
Ne I $\lambda 6074.338$ & 16.670 & -0.500 & 66 & 7.87 & 8.56 \\
Ne I $\lambda 6143.063$ & 16.620 & -0.100 & 115 & 7.84 & 8.64 \\
Ne I $\lambda 6163.594$ & 16.710 & -0.620 & 65 & 7.99 & 8.69 \\
Ne I $\lambda 6266.495$ & 16.710 & -0.370 & 97 & 7.98 & 8.77 \\
Ne I $\lambda 6334.428$ & 16.620 & -0.320 & 90 & 7.91 & 8.63 \\
Ne I $\lambda 6382.992$ & 16.670 & -0.240 & 103 & 7.89 & 8.70 \\
Ne I $\lambda 6402.246$ & 16.620 & +0.330 & 163 & 7.68 & 8.73 \\
Ne I $\lambda 6506.528$ & 16.670 & -0.030 & 121 & 7.81 & 8.68 \\
Ne I $\lambda 7032.413$ & 16.620 & -0.260 & 116 & 7.95 & 8.86 \\
Mean. & $\ldots$ & $\ldots$ & $\ldots$ & $7.87 \pm 0.10$ & $8.68 \pm 0.10$ \\
\hline
\end{tabular}

Notes.

a $\left(T_{\text {eff }}, \log g, \xi\right)=(16375,2.45,7.5)$.

${ }^{\mathrm{b}}\left(T_{\text {eff }}, \log g, \xi\right)=(17000,2.60,7.5)$.

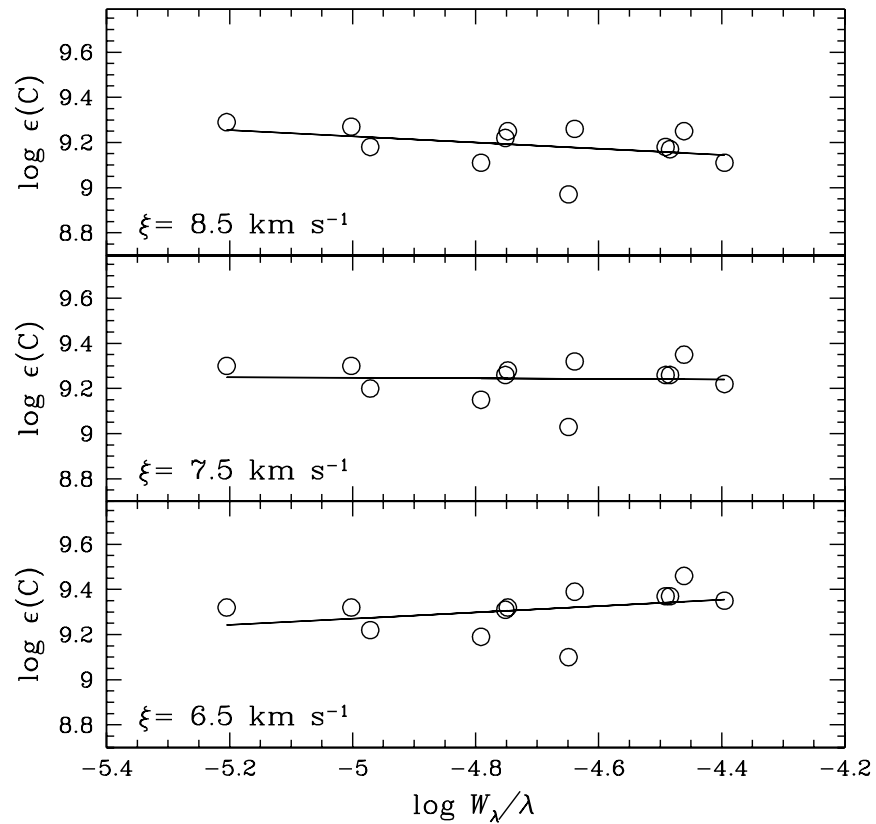

Figure 3. NLTE abundances from $\mathrm{C}_{\mathrm{II}}$ lines for $\mathrm{BD}+10^{\circ} 2179$ vs. their reduced equivalent widths $\left(\log W_{\lambda} / \lambda\right)$. A microturbulent velocity of $\xi=7.5 \mathrm{~km} \mathrm{~s}^{-1}$ is obtained from this figure.

fits to $\mathrm{He}$ I line profiles, and the ionization equilibria among $\mathrm{C}^{\circ}$, $\mathrm{C}^{+}$, and $\mathrm{C}^{2+}$. The LTE analysis is repeated but this time with TLUSTY LTE model atmospheres instead of models from the code STERNE (Jeffery et al. 2001).

Figure 3 illustrates the determination of the microturbulence from $\mathrm{C}_{\mathrm{II}}$ lines. A value of $7.5 \mathrm{~km} \mathrm{~s}^{-1}$ is adopted. Although this value is for a particular model $\left(T_{\text {eff }}=17,000 \mathrm{~K}, \log g=2.5\right)$, the result is insensitive to the model choice.

Sample theoretical NLTE line profiles and the observed profile of the $\mathrm{He}_{\mathrm{I}} 4471 \AA$ line are shown in Figure 4 for a
Table 2

Photospheric Line by Line NLTE and LTE Abundances, and the Line's Measured Equivalent Width $\left(W_{\lambda}\right)$ in $\mathrm{m} \AA$ for $\mathrm{BD}-9^{\circ} 4395$

\begin{tabular}{|c|c|c|c|c|c|}
\hline \multirow[t]{2}{*}{ Line } & \multirow{2}{*}{$\begin{array}{c}\chi \\
(\mathrm{eV})\end{array}$} & \multirow[t]{2}{*}{$\log g f$} & \multirow{2}{*}{$\begin{array}{c}W_{\lambda} \\
(\mathrm{m} \AA)\end{array}$} & \multicolumn{2}{|c|}{$\log \epsilon(\mathrm{X})$} \\
\hline & & & & NLTE $^{\mathrm{a}}$ & LTE $^{b}$ \\
\hline Н г $\lambda 3970.072$ & 10.199 & -0.993 & 60 & 9.16 & 8.90 \\
\hline Н г $\lambda 4101.734$ & 10.199 & -0.753 & 65 & 9.12 & 8.72 \\
\hline Н г $\lambda 4861.323$ & 10.199 & -0.020 & 200 & 9.10 & 8.75 \\
\hline Mean... & $\ldots$ & $\ldots$ & $\ldots$ & $9.13 \pm 0.03$ & $8.79 \pm 0.10$ \\
\hline C II $\lambda 4313.106$ & 23.116 & -0.373 & 108 & 9.27 & 9.43 \\
\hline C II $\lambda 4325.832$ & 23.119 & -0.373 & & & \\
\hline C II $\lambda 4326.164$ & 23.116 & -0.407 & 131 & 9.04 & 9.17 \\
\hline C II $\lambda 4374.281$ & 24.654 & $+0.660^{\mathrm{c}}$ & & & \\
\hline C II $\lambda 4375.008$ & 24.658 & $-0.610^{\mathrm{c}}$ & 189 & 9.01 & 9.06 \\
\hline Mean... & $\ldots$ & $\ldots$ & $\ldots$ & $9.11 \pm 0.14$ & $9.22 \pm 0.19$ \\
\hline C III $\lambda 4515.352$ & 39.401 & -0.756 & & & \\
\hline $\mathrm{C}_{\mathrm{III}} \lambda 4515.811$ & 39.402 & -0.279 & 28 & 8.92 & 8.71 \\
\hline C III $\lambda 4647.418$ & 29.535 & +0.070 & 354 & 9.04 & 9.15 \\
\hline C III $\lambda 4651.473$ & 29.535 & -0.629 & 219 & 9.23 & 9.12 \\
\hline Mean. . & $\ldots$ & $\ldots$ & $\ldots$ & $9.06 \pm 0.16$ & $8.99 \pm 0.25$ \\
\hline N II $\lambda 4601.478$ & 18.466 & -0.428 & 125 & 7.66 & 7.98 \\
\hline N II $\lambda 4607.153$ & 18.462 & -0.507 & 103 & 7.60 & 7.93 \\
\hline N II $\lambda 4621.393$ & 18.466 & -0.514 & 98 & 7.57 & 7.91 \\
\hline N II $\lambda 4630.539$ & 18.483 & +0.094 & 224 & 7.70 & 7.98 \\
\hline Mean... & $\ldots$ & $\ldots$ & $\ldots$ & $7.63 \pm 0.06$ & $7.95 \pm 0.04$ \\
\hline N III $\lambda 4103.390$ & 27.438 & -0.359 & 84 & 7.69 & 7.88 \\
\hline N III $\lambda 4195.740$ & 36.842 & -0.004 & 20 & 7.94 & 8.39 \\
\hline N III $\lambda 4200.070$ & 36.856 & +0.250 & 20 & 7.69 & 8.14 \\
\hline Mean... & . & $\ldots$ & $\ldots$ & $7.77 \pm 0.14$ & $8.14 \pm 0.26$ \\
\hline О ІІ $\lambda 4072.153$ & 25.650 & +0.552 & 134 & 8.13 & 7.78 \\
\hline O II $\lambda 4078.842$ & 25.638 & -0.284 & 32 & 7.99 & 7.77 \\
\hline O II $\lambda 4085.112$ & 25.650 & -0.189 & 46 & 8.10 & 7.86 \\
\hline О II $\lambda 4104.724$ & 25.837 & -0.302 & & & \\
\hline О II $\lambda 4104.990$ & 25.837 & -0.015 & 75 & 8.05 & 7.80 \\
\hline O II $\lambda 4303.833$ & 28.822 & $+0.640^{\mathrm{c}}$ & 40 & 7.97 & 7.67 \\
\hline O II $\lambda 4331.857$ & 28.512 & -0.136 & 42 & 8.34 & 8.41 \\
\hline О ІІ $\lambda 4336.859$ & 22.979 & -0.763 & 76 & 8.08 & 8.13 \\
\hline O II $\lambda 4342.009$ & 28.883 & $+0.820^{\mathrm{c}}$ & 44 & 7.84 & 7.51 \\
\hline O II $\lambda 4345.560$ & 22.979 & -0.346 & 119 & 7.98 & 8.00 \\
\hline O II $\lambda 4349.426$ & 22.999 & +0.060 & 212 & 8.09 & 8.10 \\
\hline O II $\lambda 4351.457$ & 25.661 & -1.004 & & & \\
\hline O II $\lambda 4351.260$ & 25.661 & +0.227 & 92 & 8.13 & 7.83 \\
\hline О ІІ $\lambda 4366.895$ & 22.999 & -0.348 & 134 & 8.08 & 8.09 \\
\hline O II $\lambda 4414.899$ & 23.442 & +0.172 & 206 & 7.86 & 8.06 \\
\hline O II $\lambda 4416.975$ & 23.419 & -0.077 & 162 & 7.91 & 8.07 \\
\hline O II $\lambda 4452.378$ & 23.442 & -0.788 & 56 & 7.92 & 8.09 \\
\hline O II $\lambda 4590.974$ & 25.661 & +0.350 & 132 & 8.38 & 8.01 \\
\hline О ІІ $\lambda 4595.957$ & 25.661 & -1.033 & & & \\
\hline O II $\lambda 4596.177$ & 25.661 & +0.200 & 109 & 8.32 & 7.99 \\
\hline О II $\lambda 4609.373$ & 29.069 & $+0.670^{\mathrm{c}}$ & 54 & 7.97 & 7.88 \\
\hline O II $\lambda 4661.632$ & 22.979 & -0.278 & 141 & 8.01 & 8.07 \\
\hline O II $\lambda 4705.346$ & 26.249 & +0.477 & 94 & 7.42 & 7.78 \\
\hline O II $\lambda 4941.072$ & 26.554 & -0.053 & 45 & 8.27 & 7.97 \\
\hline Mean... & $\ldots$ & $\ldots$ & $\ldots$ & $8.04 \pm 0.21$ & $7.95 \pm 0.20$ \\
\hline $\mathrm{Ne}_{\mathrm{I}} \lambda 5852.488$ & 16.850 & -0.490 & 21 & 8.20 & 8.65 \\
\hline $\mathrm{Ne}_{\mathrm{I}} \lambda 6143.063$ & 16.620 & -0.100 & 63 & 8.18 & 8.77 \\
\hline $\mathrm{Ne}$ I $\lambda 6266.495$ & 16.710 & -0.370 & 42 & 8.11 & 8.85 \\
\hline $\mathrm{Ne}$ I $\lambda 6334.428$ & 16.620 & -0.320 & 36 & 8.13 & 8.71 \\
\hline $\mathrm{Ne}_{\mathrm{I}} \lambda 6382.992$ & 16.670 & -0.240 & 48 & 8.17 & 8.79 \\
\hline $\mathrm{Ne}$ I $\lambda 6402.246$ & 16.620 & +0.330 & 143 & 8.19 & 8.89 \\
\hline $\mathrm{Ne}_{\mathrm{I}} \lambda 6506.528$ & 16.670 & -0.030 & 65 & 8.13 & 8.75 \\
\hline $\mathrm{Ne}$ I $\lambda 6598.953$ & 16.850 & -0.360 & 34 & 8.39 & 8.79 \\
\hline $\mathrm{Ne}$ I $\lambda 7032.413$ & 16.620 & -0.260 & 46 & 8.16 & 8.80 \\
\hline Mean... & $\ldots$ & $\ldots$ & $\ldots$ & $8.18 \pm 0.08$ & $8.78 \pm 0.07$ \\
\hline Ne II $\lambda 4379.552$ & 34.802 & +0.780 & 38 & 8.05 & 8.04 \\
\hline Ne II $\lambda 4413.113$ & 34.833 & +0.520 & 22 & 8.02 & 8.01 \\
\hline Mean... & $\ldots$ & $\ldots$ & $\ldots$ & $8.04 \pm 0.02$ & $8.03 \pm 0.02$ \\
\hline
\end{tabular}

Notes.

a $\left(T_{\text {eff }}, \log g, \xi\right)=(24300,2.65,17.5)$.

${ }^{\mathrm{b}}\left(T_{\text {eff }}, \log g, \xi\right)=(24800,2.85,23.0)$.

c Wiese et al. (1966). 


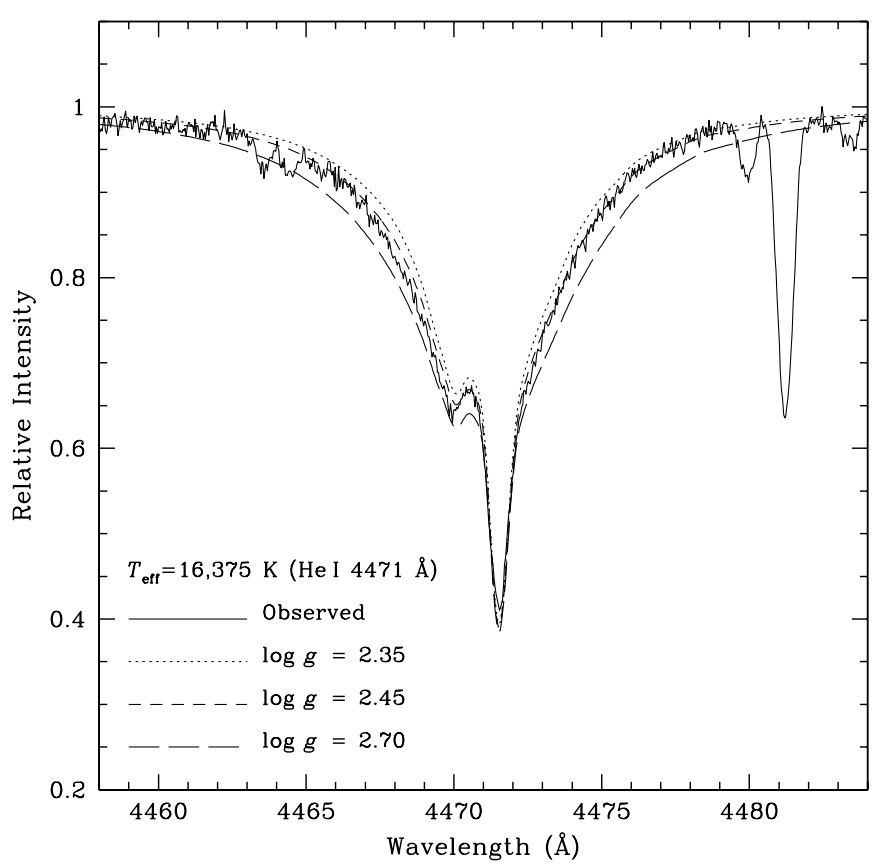

Figure 4. BD $+10^{\circ} 2179$ 's observed and synthesized NLTE He I line profile at 4471 A. The NLTE He I line profiles are synthesized using the NLTE model $T_{\text {eff }}=16,375 \mathrm{~K}$, for three different $\log g$ values—see key on the figure.

model with an effective temperature of $16,375 \mathrm{~K}$ and a surface gravity of $2.45 \mathrm{~g} \mathrm{~cm}^{-2}$ and with microturbulence and rotational broadening included (see Pandey et al. 2006). The best-fitting theoretical profile $(\log g=2.45)$ provides one point on the $T_{\text {eff }}-\log g$ locus. The He I lines at 4009, 4026, and $4387 \AA$ lines were similarly analyzed. The helium model atoms and line broadening coefficients are from TLUSTY. Using the TLUSTY grid of model atmospheres, the loci were mapped out. The four loci are shown in Figure 5 and are almost coincident.

Loci representing ionization equilibrium are provided from the requirements that $(\mathrm{C} I, C \mathrm{II}),(\mathrm{C} \mathrm{II}, \mathrm{C} I I I)$, and $\left(\mathrm{C}_{\mathrm{I}}, \mathrm{C} \mathrm{III}\right)$ provide the same $\mathrm{C}$ abundance.

Figure 5 shows the several loci. Their intersection suggests that the best NLTE model has $T_{\text {eff }}=16,375 \pm 250 \mathrm{~K}$ and $\log g=$ $2.45 \pm 0.2$. The best LTE TLUSTY model with the LTE line analysis gives a best model with $T_{\text {eff }}=17,000 \mathrm{~K}$ and $\log g=$ 2.60. This LTE model differs a little from that adopted in the 2006 LTE analysis of the optical lines where loci representing ionization equilibria for (Si II, Si III), (S II, S III), and (Fe II, Fe III) were also considered. The 2006 LTE analysis gave a model with $T_{\text {eff }}=16,400 \pm 500 \mathrm{~K}$ and $\log g=2.35 \pm 0.2 \mathrm{cgs}$.

Abundances for $\mathrm{C}, \mathrm{N}, \mathrm{O}$, and $\mathrm{Ne}$ are given in Table 1. Mean abundances and their standard deviations are listed for both the NLTE and LTE TLUSTY analyses. Abundances are given as $\log \epsilon(\mathrm{X})$ and normalized to $\log \Sigma \mu_{\mathrm{X}} \epsilon(\mathrm{X})=12.15$, where $\mu_{\mathrm{X}}$ is the atomic weight of element X. The NLTE abundance errors arising from uncertainties in the atmospheric parameters are estimated by considering changes of $\Delta T= \pm 250 \mathrm{~K}, \Delta \log g=$ $\pm 0.2 \mathrm{cgs}$, and $\Delta \xi= \pm 1 \mathrm{~km} \mathrm{~s}^{-1}$. The rms errors in the abundances from $\mathrm{C}_{\mathrm{I}}, \mathrm{C}$ II, C $\mathrm{III}, \mathrm{N}$ II, O II, and $\mathrm{Ne}$ I are 0.22 , $0.03,0.18,0.08,0.12$, and 0.10 , respectively, with a negligible contribution from the microturbulence. The $\mathrm{C} / \mathrm{He}$ ratio is $0.6 \%$ but a ratio of $1 \%$ was assumed in construction of the NLTE model. Recomputation of the model for $\mathrm{C} / \mathrm{He}=0.6 \%$ results in negligible changes to the abundances in Table 1. Abundance uncertainties are similar for the LTE analysis.

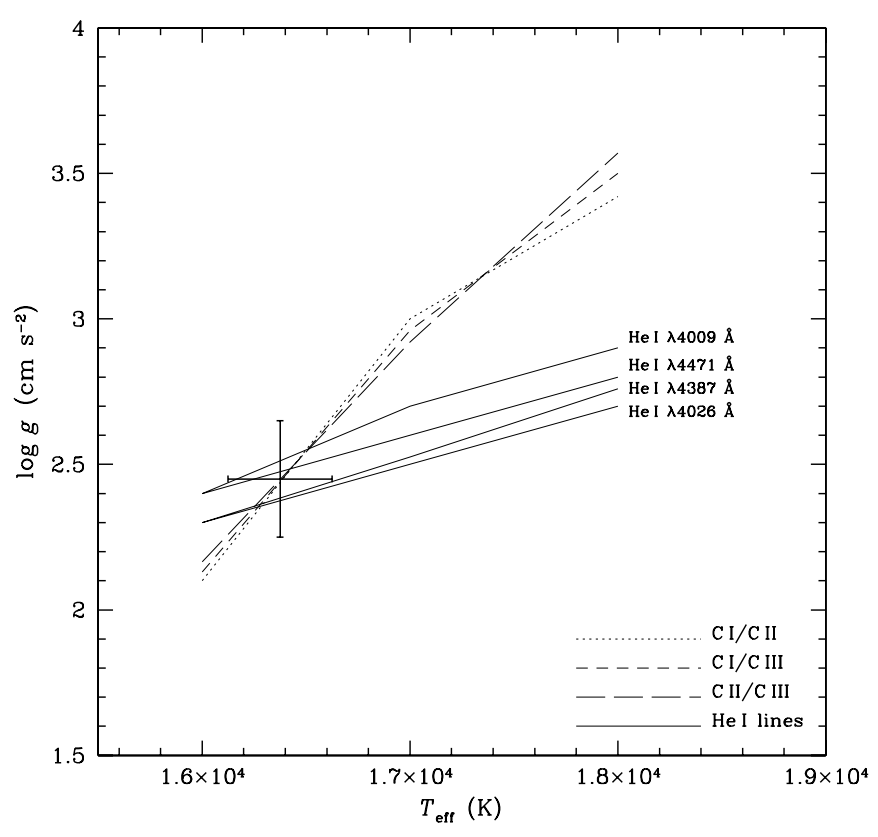

Figure 5. $T_{\text {eff }}$ vs. $\log g$ plane for $\mathrm{BD}+10^{\circ} 2179$. Loci satisfying ionization equilibria are plotted-see keys on the figure. The loci satisfying optical He I line profiles $(\lambda 4471,4387,4026$, and $4009 \AA)$ are shown by the solid lines. The cross shows the adopted NLTE model atmosphere parameters.

With the exception of $\mathrm{HI}, \mathrm{C}$ III, and $\mathrm{Ne}$ I lines, the introduction of NLTE for the model atmosphere and line analysis has a minor effect on the derived abundances. The mean abundance differences in dex in the sense (LTE-NLTE) are 0.07 (C I), 0.04 (C II), -0.07 (N II), -0.26 (O II), and 0.81 (Ne I).

The H I lines show similar NLTE effects (LTE-NLTE) across the lines. The difference in abundance (LTE-NLTE) is about 0.33 dex. Note that the NLTE/LTE abundance from $\mathrm{H} \beta$ down the sequence decreases by about 0.3 dex.

The $\mathrm{C}$ III lines represent a fascinating issue in line formation. In the LTE analysis, the $4186.9 \AA 40 \mathrm{eV}$ line gives an abundance that is 0.6 dex greater than that from the $4650 \AA$ triplet which provides a more plausible abundance. In NLTE, however, the abundance discrepancy is reversed: the $4186 \AA$ line gives a plausible abundance that is 0.7 dex less than that from the triplet. Nieva \& Przybilla (2008) state that the sense of this reversal is expected according to their calculations for normal B stars. The magnitude of the NLTE effects and the failure of our calculations to provide consistent NLTE abundances suggest that the $\mathrm{C}$ III be given lower weight in the analysis.

There are small and unimportant differences between the 2006 LTE abundances and those in Table 1. Such differences arise from a combination of factors: the model atmosphere codes are different, and the derived atmospheric parameters are different. The differences in dex in the sense (TLUSTY-STERNE) are 0.12 (C I ), 0.04 (C II), 0.14 (N II), 0.18 (O II), and 0.04 (Ne I).

\section{2. $B D-9^{\circ} 4395$}

This star's spectrum contains absorption lines with variable profiles and variable emission lines mainly from $\mathrm{He}$ I, $\mathrm{C}$ II, and $\mathrm{Si}$ II transitions. These emission lines have been attributed to a shell or extended atmosphere. An extensive library of optical and ultraviolet spectra of BD-9 ${ }^{\circ} 4395$ was discussed by Jeffery \& Heber (1992) who undertook an abundance analysis using absorption lines drawn from a mean optical spectrum. Their LTE analysis led to the atmospheric parameters: $T_{\text {eff }}=22,700 \pm$ 


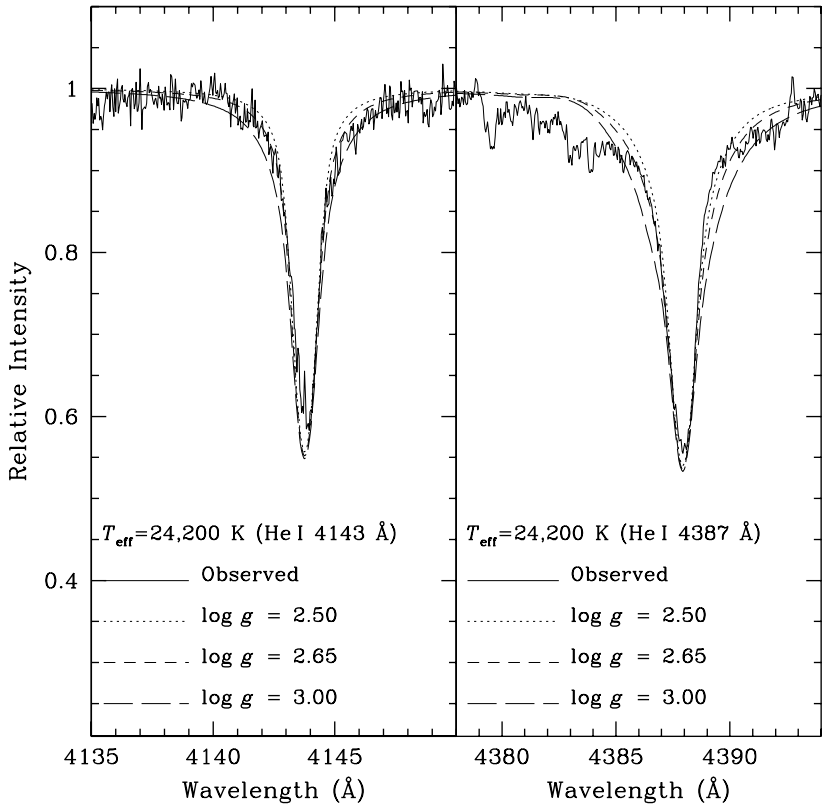

Figure 6. BD $-9^{\circ} 4395$ 's observed and synthesized NLTE He I line profiles at $4143 \AA$ and at $4387 \AA$. The NLTE He I line profiles are synthesized using the NLTE model $T_{\text {eff }}=24,200 \mathrm{~K}$, for three different $\log g$ values-see key on the figure.

$1200 \mathrm{~K}, \log g=2.55 \pm 0.10$, and $\xi=20 \pm 5 \mathrm{~km} \mathrm{~s}^{-1}$. In addition to the line broadening from the high microturbulence and line profile variations, the line profiles suggested that the star may be rotating at about $40 \mathrm{~km} \mathrm{~s}^{-1}$.

Our high-resolution optical spectra confirm the characteristics described by Jeffery \& Heber. We measure equivalent widths off our spectra. Most of the measured equivalent widths are from the 2000 June 16 spectrum. These measured equivalent widths are in fair agreement with those measured off the spectra obtained on other dates.

Our abundance analysis follows the method discussed in the previous section. Details about the majority of the lines are taken from Pandey et al. (2006) with information on other lines of C II-III, N II-III, O II, and Ne II from the NIST database (http://physics.nist.gov/PhysRefData/ASD/lines_form.html).

The source of $g f$-values for $\mathrm{Ne}$ I lines is as given in Section 4.1.

The $\mathrm{O}$ II lines confirm the high microturbulence with our NLTE analyses giving $\xi=17.5 \pm 5 \mathrm{~km} \mathrm{~s}^{-1}$. This value is not sensibly different from the $20 \mathrm{~km} \mathrm{~s}^{-1}$ obtained by Jeffery \& Heber. The microturbulence is somewhat higher than that found for most other EHe stars and indicates supersonic atmospheric motions.

The He I lines are moderately sensitive to gravity. As clearly noted by Jeffery \& Heber, emission affects the He I profiles to differing degrees. For example, the $5876 \AA$ line is in emission. Observed profiles of the $4143 \AA$ and $4387 \AA$ lines are shown in Figure 6 with predicted NLTE profiles for an NLTE atmosphere of $T_{\text {eff }}=24,300 \mathrm{~K}$ and three different surface gravities. The predicted profiles have been convolved with a (Gaussian) profile with an FWHM of $40 \mathrm{~km} \mathrm{~s}^{-1}$ to represent the projected rotational velocity suggested by Jeffery \& Heber. The chosen lines are those least affected by emission (Jeffery \& Heber 1992). There may be indications that weak emission contaminates the red wing and, perhaps, the line core. LTE profiles shown by Jeffery \& Heber predict less deep cores than the observed profiles; the NLTE profiles reproduce the line cores more closely than LTE profiles.

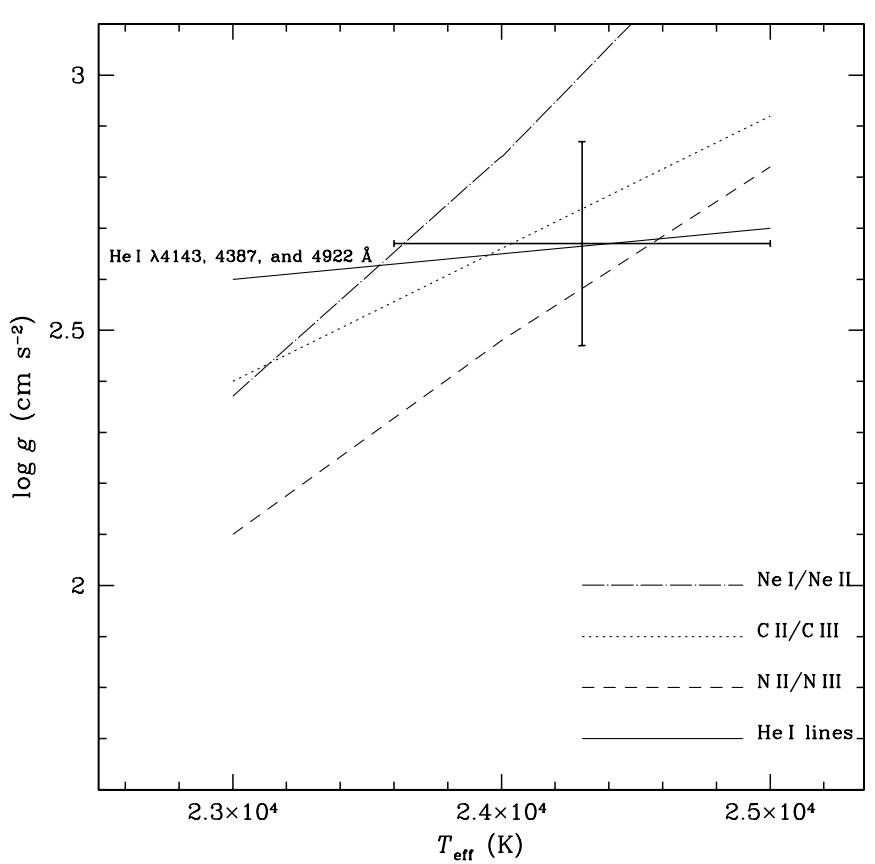

Figure 7. $T_{\text {eff }}$ vs. $\log g$ plane for BD-9 ${ }^{\circ} 4395$. Loci satisfying ionization equilibria are plotted-see keys on the figure. The locus satisfying optical He I line profiles $(\lambda 4143,4387$, and $4922 \AA$ ) is shown by the solid line. The cross shows the adopted NLTE model atmosphere parameters.

Ionization equilibria $\mathrm{C}$ II/C III and $\mathrm{N}$ II/N III provide two loci in the $\left(T_{\text {eff }}, \log g\right)$ plane (Figure 7$)$. Inspection of this figure suggests a solution with $T_{\text {eff }}=24,300 \pm 700 \mathrm{~K}$ and $\log g=$ $2.65 \pm 0.20 \mathrm{cgs}$, where we give equal weight to the $\mathrm{C}$ and $\mathrm{N}$ ionization equilibria. This effective temperature is $1600 \mathrm{~K}$ hotter than that estimated by Jeffery \& Heber. The difference is partly accounted for by the fact that the earlier (LTE) analysis included loci representing ionization equilibrium for Si II/Si IV and S II/S III and these loci of similar slope to the $\mathrm{C}$ and $\mathrm{N}$ loci fell about $1000 \mathrm{~K}$ to lower temperatures. Final abundances for our adopted model are given in Table 2. Mean abundances and their standard deviations are given. The rms uncertainties arising from the estimated uncertainties of the atmospheric parameters are 0.05 (C II), 0.16 (C III), 0.08 (N II), 0.20 (N III), 0.02 (O II), 0.08 ( Ne I), and 0.16 ( Ne II).

The LTE abundances in Table 2 were computed from a TLUSTY LTE model atmosphere with model parameters $\left(T_{\text {eff }}\right.$, $\log g, \xi)=(24,800,2.85,23.0)$. Line-by-line LTE abundances including the mean abundance and the line-to-line scatter are given in Table 2. These LTE abundances are quite similar to those reported by Jeffery \& Heber from a different line list with different atomic data, a different model chosen from a different grid of LTE atmospheres: the differences in dex in the sense (TLUSTY-JH) are 0.22 (C II), -0.35 (C III), 0.03 ( $\mathrm{N}$ II), -0.01 ( $\mathrm{N}$ III), 0.05 (O II), 0.02 (Ne I), and -0.13 (Ne II).

Corrections for NLTE effects in the sense (LTE-NLTE) are -0.34 (H I), 0.11 (C II), -0.07 (C III), 0.32 (N II), 0.37 N III, -0.09 (O II), 0.60 ( Ne I), and -0.01 (Ne II) in dex. In the case of $\mathrm{Ne}$, the two stages of ionization treated in NLTE give consistent abundances but do not in LTE. Also noteworthy is that the C III lines treated in NLTE give fairly consistent results but this was not the case for $\mathrm{BD}+10^{\circ} 2179$.

The NLTE correction (LTE-NLTE) for $\mathrm{H}_{\mathrm{I}}$ is about -0.34 dex, a reversal in the NLTE correction that was provided by the analysis of $\mathrm{BD}+10^{\circ} 2179$. It appears that the NLTE 
Table 3

Photospheric Line by Line LTE Neon Abundances, NLTE Neon Abundance, and the Line's Measured Equivalent Width $\left(W_{\lambda}\right)$ in $\mathrm{m} \AA$ for LSE 78

\begin{tabular}{|c|c|c|c|c|c|}
\hline \multirow[t]{2}{*}{ Line } & \multirow{2}{*}{$\begin{array}{c}\chi \\
(\mathrm{eV})\end{array}$} & \multirow[t]{2}{*}{$\log g f$} & \multirow{2}{*}{$\begin{array}{c}W_{\lambda} \\
(\mathrm{m} \AA) \\
\end{array}$} & \multicolumn{2}{|c|}{$\log \epsilon(\mathrm{Ne})$} \\
\hline & & & & NLTE $^{\mathrm{a}}$ & $\mathrm{LTE}^{\mathrm{b}}$ \\
\hline $\mathrm{Ne}_{\mathrm{I}} \lambda 5852.488$ & 16.850 & -0.490 & 155 & & 9.36 \\
\hline $\mathrm{Ne}_{\mathrm{I}} \lambda 5881.895$ & 16.620 & -0.770 & 128 & & 9.46 \\
\hline Ne I $\lambda 6029.997$ & 16.670 & -1.040 & 81 & & 9.49 \\
\hline $\mathrm{Ne}_{\mathrm{I}} \lambda 6074.338$ & 16.670 & -0.500 & 159 & & 9.37 \\
\hline $\mathrm{Ne}_{\mathrm{I}} \lambda 6143.063$ & 16.620 & -0.100 & 285 & & 9.52 \\
\hline $\mathrm{Ne}$ I $\lambda 6163.594$ & 16.710 & -0.620 & 137 & & 9.40 \\
\hline $\mathrm{Ne}_{\mathrm{I}} \lambda 6217.281$ & 16.620 & -0.960 & 84 & & 9.42 \\
\hline $\mathrm{Ne}_{\mathrm{I}} \lambda 6266.495$ & 16.710 & -0.370 & 222 & & 9.55 \\
\hline $\mathrm{Ne}$ I $\lambda 6334.428$ & 16.620 & -0.320 & 241 & & 9.57 \\
\hline Ne I $\lambda 6382.992$ & 16.670 & -0.240 & 254 & & 9.56 \\
\hline $\mathrm{Ne}$ I $\lambda 6402.246$ & 16.620 & +0.330 & 409 & & 9.59 \\
\hline $\mathrm{Ne}$ I $\lambda 6506.528$ & 16.670 & -0.030 & 287 & & 9.49 \\
\hline $\mathrm{Ne}_{\mathrm{I}} \lambda 6532.882$ & 16.710 & -0.720 & 123 & & 9.47 \\
\hline $\mathrm{Ne}_{\mathrm{I}} \lambda 6598.953$ & 16.850 & -0.360 & 119 & & 9.11 \\
\hline Ne I $\lambda 6717.043$ & 16.850 & -0.360 & 138 & & 9.22 \\
\hline $\mathrm{Ne}$ I $\lambda 7032.413$ & 16.620 & -0.260 & 229 & & 9.47 \\
\hline Mean... & $\ldots$ & $\ldots$ & $\ldots$ & 8.67 & $9.40 \pm 0.13$ \\
\hline
\end{tabular}

Notes.

a By applying correction on the LTE neon abundance.

${ }^{\mathrm{b}}\left(T_{\mathrm{eff}}, \log g, \xi\right)=(18300,2.20,16.0)$.

correction (LTE-NLTE) is mainly a function of effective temperature as these stars are of similar surface gravity.

\subsection{LSE 78, V1920 Cyg, HD 124448, and PV Tel}

Neon abundances for this quartet are estimated by applying corrections to the LTE Ne abundances from Ne I lines based on the NLTE calculations computed for model atmosphere grids computed for $\mathrm{BD}+10^{\circ} 2179$ and $\mathrm{BD}-9^{\circ}$ 4395. NLTE Ne abundances for LSE 78 and V1920 Cyg are estimated by interpolation in the grids of computed NLTE corrections but for HD 124448 and PV Tel an extrapolation is required. Neon LTE abundances are computed with the LTE models and the Armagh LTE code SPECTRUM (Jeffery \& Heber 1992; Jeffery et al. 2001). In Tables 3-6, we give line-by-line LTE neon abundances including the mean abundance, and the line-to-line scatter. The estimated NLTE corrections to the LTE neon abundances of LSE 78, V1920 Cyg, HD 124448, and PV Tel are 0.73, 0.8, 0.8, and 0.88 , respectively.

For LSE 78 and V1920 Cyg, the LTE Ne abundance is independent of an Ne I's line equivalent width when the microturbulence from the 2006 paper is adopted. For HD 124448, two weak $\mathrm{Ne}$ I lines provide the abundance and the adopted value of the microturbulence is unimportant. In the case of PV Tel, the only $\mathrm{Ne}$ I lines available from our spectra are strong and the microturbulence from the 2006 paper gives an $\mathrm{Ne}$ abundance that is a function of a line's equivalent width, a trend that may be removed by increasing the adopted value of the microturbulence from the $15 \pm 4 \mathrm{~km} \mathrm{~s}^{-1}$ found in 2006 from optical N II and $\mathrm{S}$ II lines to $25 \mathrm{~km} \mathrm{~s}^{-1}$ and then the LTE Ne abundance is $8.53 \pm 0.08$ (Table 6). An estimated NLTE correction of $0.9 \mathrm{dex}$ gives the NLTE Ne abundance of 7.6.

\section{4. $L S I V+6^{\circ} 002$}

Abundance analysis of LS IV $+6^{\circ} 002$ was done by Jeffery (1998) using absorption line equivalent widths drawn from the optical spectrum. This LTE analysis led to the atmospheric
Table 4

Photospheric Line by Line LTE Neon Abundances, NLTE Neon Abundance, and the Line's Measured Equivalent Width $\left(W_{\lambda}\right)$ in $\mathrm{m} \AA$ for V1920, Cyg

\begin{tabular}{|c|c|c|c|c|c|}
\hline \multirow[t]{2}{*}{ Line } & \multirow{2}{*}{$\begin{array}{c}\chi \\
(\mathrm{eV})\end{array}$} & \multirow[t]{2}{*}{$\log g f$} & \multirow{2}{*}{$\begin{array}{c}W_{\lambda} \\
(\mathrm{m} \AA)\end{array}$} & \multicolumn{2}{|c|}{$\log \epsilon(\mathrm{Ne})$} \\
\hline & & & & NLTE $^{\mathrm{a}}$ & $\mathrm{LTE}^{\mathrm{b}}$ \\
\hline $\mathrm{Ne}_{\mathrm{I}} \lambda 5852.488$ & 16.850 & -0.490 & 185 & & 9.18 \\
\hline $\mathrm{Ne}_{\mathrm{I}} \lambda 5881.895$ & 16.620 & -0.770 & 204 & & 9.47 \\
\hline Ne I $\lambda 6029.997$ & 16.670 & -1.040 & 114 & & 9.38 \\
\hline $\mathrm{Ne}$ I $\lambda 6074.338$ & 16.670 & -0.500 & 230 & & 9.34 \\
\hline $\mathrm{Ne}$ I $\lambda 6143.063$ & 16.620 & -0.100 & 359 & & 9.44 \\
\hline Ne I $\lambda 6163.594$ & 16.710 & -0.620 & 198 & & 9.35 \\
\hline $\mathrm{Ne}$ I $\lambda 6217.281$ & 16.620 & -0.960 & 93 & & 9.18 \\
\hline Ne I $\lambda 6266.495$ & 16.710 & -0.370 & 264 & & 9.38 \\
\hline Ne I $\lambda 6334.428$ & 16.620 & -0.320 & 258 & & 9.28 \\
\hline $\mathrm{Ne}$ I $\lambda 6382.992$ & 16.670 & -0.240 & 319 & & 9.45 \\
\hline $\mathrm{Ne}$ I $\lambda 6402.246$ & 16.620 & +0.330 & 472 & & 9.47 \\
\hline Ne I $\lambda 6506.528$ & 16.670 & -0.030 & 373 & & 9.46 \\
\hline Ne I $\lambda 6532.882$ & 16.710 & -0.720 & 176 & & 9.40 \\
\hline Ne I $\lambda 6598.953$ & 16.850 & -0.360 & 177 & & 9.07 \\
\hline Ne I $\lambda 6717.043$ & 16.850 & -0.360 & 177 & & 9.09 \\
\hline $\mathrm{Ne}$ I $\lambda 7032.413$ & 16.620 & -0.260 & 340 & & 9.55 \\
\hline Mean... & $\ldots$ & $\ldots$ & $\ldots$ & 8.50 & $9.30 \pm 0.14$ \\
\hline
\end{tabular}

Notes.

a By applying correction on the LTE neon abundance.

${ }^{\mathrm{b}}\left(T_{\mathrm{eff}}, \log g, \xi\right)=(16330,1.80,20.0)$.

Table 5

Photospheric Line by Line LTE Neon Abundances, NLTE Neon Abundance, and the Line's Measured Equivalent Width $\left(W_{\lambda}\right)$ in $\mathrm{m} \AA$ for HD 124448

\begin{tabular}{lccccc}
\hline \hline \multicolumn{1}{c}{ Line } & $\chi$ & $\log g f$ & $W_{\lambda}$ & \multicolumn{2}{c}{$\log \epsilon(\mathrm{Ne})$} \\
\cline { 5 - 6 } & $(\mathrm{eV})$ & & $(\mathrm{m} \AA)$ & NLTE $^{\mathrm{a}}$ & LTE $^{\mathrm{b}}$ \\
\hline Ne I $\lambda 6334.428$ & 16.620 & -0.320 & 118 & & 8.45 \\
Ne I $\lambda 6717.043$ & 16.850 & -0.360 & 105 & & 8.51 \\
Mean. & $\ldots$ & $\ldots$ & $\ldots$ & 7.70 & $8.50 \pm 0.04$ \\
\hline
\end{tabular}

Notes.

a By applying correction on the LTE neon abundance.

${ }^{\mathrm{b}}\left(T_{\mathrm{eff}}, \log g, \xi\right)=(15500,1.90,12.0)$.

Table 6

Photospheric Line by Line LTE Neon Abundances, NLTE Neon Abundance, and the Line's Measured Equivalent Width $\left(W_{\lambda}\right)$ in $\mathrm{m} \AA$ for PV Tel

\begin{tabular}{cccccc}
\hline \hline \multicolumn{1}{c}{ Line } & $\chi$ & $\log g f$ & $W_{\lambda}$ & \multicolumn{2}{c}{$\log \epsilon(\mathrm{Ne})$} \\
\cline { 5 - 6 } & $(\mathrm{eV})$ & & $(\mathrm{m} \AA)$ & NLTE $^{\mathrm{a}}$ & LTE $^{\mathrm{b}}$ \\
\hline $\mathrm{Ne}_{\mathrm{I}} \lambda 5881.895$ & 16.620 & -0.770 & 233 & & 8.52 \\
$\mathrm{Ne}_{\mathrm{I}} \lambda 6074.338$ & 16.670 & -0.500 & 324 & & 8.53 \\
$\mathrm{Ne}_{\mathrm{I}} \lambda 6096.163$ & 16.670 & -0.310 & 354 & & 8.42 \\
$\mathrm{Ne}_{\mathrm{I}} \lambda 6402.246$ & 16.620 & +0.330 & 671 & & 8.70 \\
Ne I $\lambda 7032.413$ & 16.620 & -0.260 & 422 & & 8.58 \\
Mean... & $\ldots$ & $\ldots$ & $\ldots$ & 7.60 & $8.53 \pm 0.08$ \\
\hline
\end{tabular}

Notes.

a By applying correction on the LTE neon abundance.

${ }^{\mathrm{b}}\left(T_{\text {eff }}, \log g, \xi\right)=(13750,1.60,25.0)$.

parameters: $T_{\text {eff }}=31,800 \pm 800 \mathrm{~K}, \log g=4.05 \pm 0.10$, and $\xi=9 \pm 1 \mathrm{~km} \mathrm{~s}^{-1}$. This is the hottest star in our sample with $\mathrm{Ne}$ II but not Ne I lines in its spectrum.

Here the Jeffery (1998) equivalent widths have been reanalyzed using our $g f$-values from Pandey et al. (2006) and the NIST database. Two sets of model atmospheres are considered: NLTE/TLUSTY and LTE/TLUSTY. Analyses of the C III, N II, and $\mathrm{O}$ II lines confirm the microturbulence obtained by Jeffery 


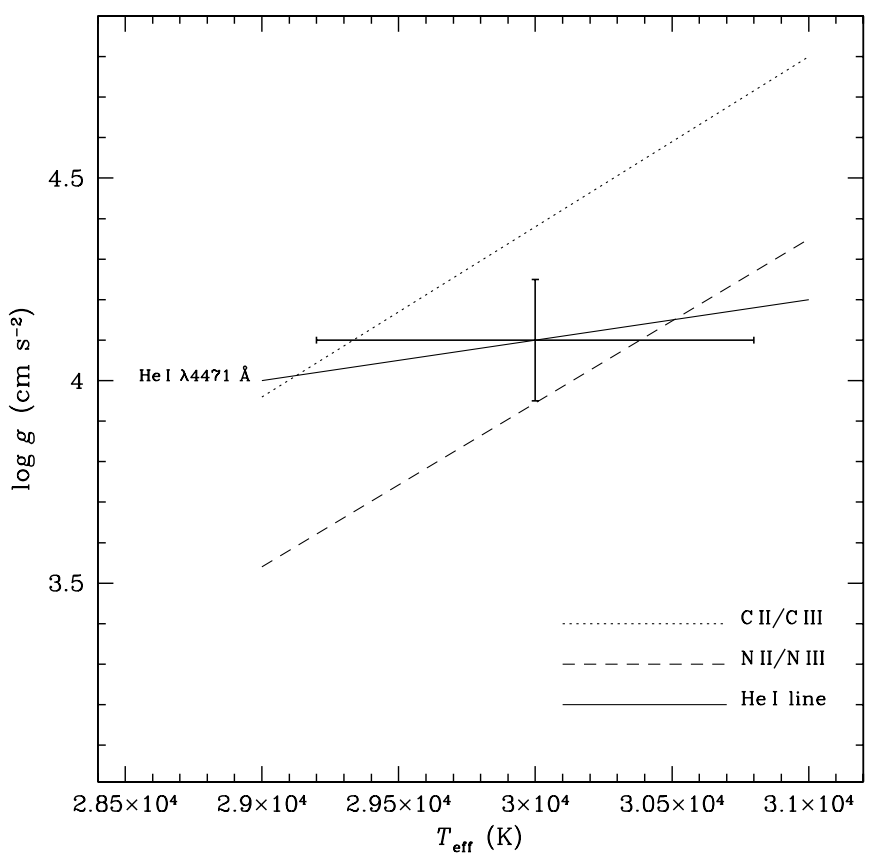

Figure 8. $T_{\text {eff }}$ vs. $\log g$ plane for $\operatorname{LS}$ IV $+6^{\circ}$ 002. Loci satisfying ionization equilibria are plotted-see keys on the figure. The locus satisfying the optical He I $4471 \AA$ line profile is shown by the solid line. The cross shows the adopted NLTE model atmosphere parameters.

with our NLTE and LTE analyses giving $\xi$ about $9 \mathrm{~km} \mathrm{~s}^{-1}$. Ionization equilibria $\mathrm{C}$ II/C III, and $\mathrm{N}$ II/N III provide two loci in the $\left(T_{\text {eff }}, \log g\right.$ ) plane (Figure 8$)$. The He I $4471 \AA$ line that is moderately insensitive to gravity provides another locus.

Inspection of Figure 8, produced by adopting NLTE/ TLUSTY models, suggests a solution with $T_{\text {eff }}=30,000 \pm$ $800 \mathrm{~K}$ and $\log g=4.10 \pm 0.15 \mathrm{cgs}$. The He II $4686 \AA$ line suggests an effective temperature about 1000-2000 K hotter. Here we give more weight to the $\mathrm{C}$ and $\mathrm{N}$ ionization equilibria, and the locus provided by the He I $4471 \AA$ line. This effective temperature is $2000 \mathrm{~K}$ cooler than that estimated by Jeffery. Final abundances for our adopted model are given in Table 7. Mean abundances and their standard deviations are given. Corrections for NLTE effects in the sense (LTE-NLTE) in dex are as follows: -0.33 (H I), 0.13 (C II), -0.36 (C III), -0.30 (N II), 0.25 N III, 0.14 (O II), and -0.02 (Ne II).

The LTE abundances in Table 7 were computed from a TLUSTY LTE model atmosphere. The best TLUSTY LTE model parameters are $\left(T_{\text {eff }}, \log g, \xi\right)=(32,000,4.20,9.0)$. Note that no weight is given to the $\mathrm{C}$ ionization equilibrium suspecting departures from LTE. Line by line LTE abundances including the mean abundance and the line-to-line scatter are given in Table 7. These LTE abundances are quite similar to those reported by Jeffery from a different line list with different atomic data, a different model chosen from a different grid of LTE atmospheres.

Our NLTE Ne abundance in Table 7 is based on Kurucz $g f$ values for the Ne II lines. The NLTE corrections for these Ne II lines are small, being typically $0.02 \mathrm{dex}$ in the sense that the NLTE abundance is higher than the LTE value. Jeffery's 1998 LTE Ne abundance is based on $g f$-values that are systematically smaller than our adopted values with a mean difference of 0.7 dex. Thus, our LTE Ne abundance is 0.7 dex lower than Jeffery's value of 9.33 .
Table 7

Photospheric Line by Line NLTE and LTE Abundances, and the Line's Measured Equivalent Width $\left(W_{\lambda}\right)$ in $\mathrm{m} \AA$ for $\operatorname{LS~IV~}+6^{\circ} 002$

\begin{tabular}{|c|c|c|c|c|c|}
\hline \multirow[t]{2}{*}{ Line } & \multirow{2}{*}{$\begin{array}{c}\chi \\
(\mathrm{eV})\end{array}$} & \multirow[t]{2}{*}{$\log g f$} & \multirow{2}{*}{$\begin{array}{c}W_{\lambda} \\
(\mathrm{m} \AA)\end{array}$} & \multicolumn{2}{|c|}{$\log \epsilon(\mathrm{X})$} \\
\hline & & & & $\mathrm{NLTE}^{\mathrm{a}}$ & $\mathrm{LTE}^{\mathrm{b}}$ \\
\hline $\mathrm{H} \mathrm{I} \lambda 4340.462$ & 10.199 & -0.447 & 20 & 8.15 & 7.95 \\
\hline Н г $\lambda 4861.323$ & 10.199 & -0.020 & 11 & 7.80 & 7.34 \\
\hline Mean... & $\ldots$ & $\ldots$ & $\ldots$ & $7.98 \pm 0.25$ & $7.65 \pm 0.43$ \\
\hline C II $\lambda 4267.001$ & 18.046 & +0.563 & & & \\
\hline $\mathrm{C}_{\text {II }} \lambda 4267.183$ & 18.046 & +0.716 & & & \\
\hline C II $\lambda 4267.261$ & 18.046 & -0.584 & 331 & $8.70 ?$ & $8.90 ?$ \\
\hline $\mathrm{C}_{\text {II }} \lambda 4285.703$ & 24.602 & $-0.430^{\mathrm{c}}$ & 61 & 9.42 & 9.57 \\
\hline C II $\lambda 4291.815$ & 24.603 & $-0.500^{\mathrm{d}}$ & & & \\
\hline $\mathrm{C}_{\text {II }} \lambda 4291.858$ & 24.603 & $-0.500^{\mathrm{d}}$ & 107 & 9.67 & $10.18 ?$ \\
\hline $\mathrm{C}_{\text {II }} \lambda 4313.106$ & 23.116 & -0.373 & 85 & 9.31 & 9.50 \\
\hline C II $\lambda 4317.265$ & 23.119 & -0.005 & 147 & 9.53 & 9.72 \\
\hline C II $\lambda 4318.606$ & 23.114 & -0.407 & 64 & 9.12 & 9.30 \\
\hline C II $\lambda 4372.375$ & 24.656 & $+0.057^{\mathrm{c}}$ & 162 & $9.96 ?$ & $10.12 ?$ \\
\hline C II $\lambda 4413.271$ & 24.603 & -0.610 & 67 & 9.70 & 9.85 \\
\hline Mean. .. & $\ldots$ & $\ldots$ & $\ldots$ & $9.46 \pm 0.22$ & $9.59 \pm 0.21$ \\
\hline C III $\lambda 4067.940$ & 39.923 & +0.720 & 154 & 9.25 & 8.85 \\
\hline $\mathrm{C}_{\text {III }} \lambda 4068.916$ & 39.924 & +0.838 & & & \\
\hline C III $\lambda 4068.916$ & 39.924 & -0.340 & 155 & 9.11 & 8.71 \\
\hline $\mathrm{C}_{\text {III }} \lambda 4070.260$ & 39.925 & +0.953 & & & \\
\hline C III $\lambda 4070.306$ & 39.925 & -0.339 & 170 & 9.10 & 8.70 \\
\hline C III $\lambda 4186.900$ & 40.010 & +0.918 & 181 & 9.29 & 8.88 \\
\hline C III $\lambda 4647.418$ & 29.535 & +0.070 & 299 & 9.24 & 9.08 \\
\hline C III $\lambda 4650.246$ & 29.535 & -0.151 & 262 & 9.30 & 9.10 \\
\hline $\mathrm{C}_{\text {III }} \lambda 4651.473$ & 29.535 & -0.629 & 168 & 9.21 & 8.85 \\
\hline C III $\lambda 4659.058$ & 38.218 & -0.654 & 59 & 9.60 & 9.18 \\
\hline C III $\lambda 4663.642$ & 38.219 & -0.530 & 63 & 9.53 & 9.12 \\
\hline C III $\lambda 4665.860$ & 38.226 & +0.044 & 101 & 9.40 & 9.01 \\
\hline C III $\lambda 4673.953$ & 38.226 & -0.433 & 69 & 9.52 & 9.11 \\
\hline Mean.... & & $\ldots$ & $\ldots$ & $9.32 \pm 0.17$ & $8.96 \pm 0.17$ \\
\hline N II $\lambda 3994.997$ & 18.497 & +0.208 & 139 & 7.88 & 8.34 \\
\hline $\mathrm{N}_{\text {II }} \lambda 4035.081$ & 23.124 & $+0.623^{\mathrm{e}}$ & 97 & 8.01 & 8.27 \\
\hline $\mathrm{N}_{\text {II }} \lambda 4041.310$ & 23.142 & $+0.853^{\mathrm{e}}$ & 129 & 8.10 & 8.37 \\
\hline $\mathrm{N}_{\text {II }} \lambda 4043.532$ & 23.132 & $+0.743^{\mathrm{e}}$ & 92 & 7.84 & 8.10 \\
\hline N II $\lambda 4044.779$ & 23.132 & $-0.437^{\mathrm{e}}$ & 66 & 8.74 & 8.99 \\
\hline $\mathrm{N}_{\text {II }} \lambda 4056.907$ & 23.142 & $-0.437^{\mathrm{e}}$ & 49 & 8.53 & 8.78 \\
\hline N II $\lambda 4073.053$ & 23.124 & $-0.160^{\mathrm{d}}$ & 62 & 8.42 & 8.67 \\
\hline $\mathrm{N}_{\text {II }} \lambda 4082.270$ & 23.132 & $+0.150^{\mathrm{d}}$ & 70 & 8.21 & 8.46 \\
\hline N II $\lambda 4173.561$ & 23.242 & $-0.570^{\mathrm{d}}$ & 42 & 8.60 & 8.85 \\
\hline N II $\lambda 4179.674$ & 23.246 & $-0.090^{\mathrm{d}}$ & 55 & 8.30 & 8.55 \\
\hline $\mathrm{N}_{\text {II }} \lambda 4236.927$ & 23.239 & $+0.383^{\mathrm{e}}$ & & & \\
\hline N II $\lambda 4237.047$ & 23.242 & $+0.553^{\mathrm{d}}$ & 109 & 7.93 & 8.18 \\
\hline $\mathrm{N}_{\text {II }} \lambda 4427.233$ & 23.422 & $-0.010^{\mathrm{d}}$ & 58 & 8.33 & 8.57 \\
\hline N II $\lambda 4427.963$ & 23.422 & $-0.170^{\mathrm{e}}$ & 60 & 8.50 & 8.74 \\
\hline $\mathrm{N}_{\text {II }} \lambda 4431.814$ & 23.415 & $-0.170^{\mathrm{e}}$ & 47 & 8.33 & 8.57 \\
\hline $\mathrm{N}_{\text {II }} \lambda 4432.736$ & 23.415 & $+0.580^{\mathrm{e}}$ & 92 & 8.09 & 8.34 \\
\hline $\mathrm{N}_{\text {II }} \lambda 4433.475$ & 23.425 & $-0.040^{\mathrm{e}}$ & 58 & 8.35 & 8.58 \\
\hline $\mathrm{N}_{\text {II }} \lambda 4442.015$ & 23.422 & $+0.310^{\mathrm{e}}$ & 50 & 7.89 & 8.13 \\
\hline $\mathrm{N}_{\text {II }} \lambda 4447.030$ & 20.409 & +0.228 & 110 & 8.03 & 8.40 \\
\hline $\mathrm{N}_{\text {II }} \lambda 4530.410$ & 23.475 & $+0.670^{\mathrm{e}}$ & 103 & 8.16 & 8.41 \\
\hline N II $\lambda 4601.478$ & 18.466 & -0.428 & 112 & 8.30 & 8.74 \\
\hline N II $\lambda 4607.153$ & 18.462 & -0.507 & 118 & 8.45 & 8.89 \\
\hline N II $\lambda 4613.868$ & 18.466 & -0.665 & 93 & 8.34 & 8.76 \\
\hline N II $\lambda 4621.393$ & 18.466 & -0.514 & 122 & 8.50 & 8.94 \\
\hline $\mathrm{N}_{\text {II }} \lambda 4630.539$ & 18.483 & +0.094 & 173 & 8.45 & 8.97 \\
\hline N II $\lambda 4678.135$ & 23.572 & $+0.434^{\mathrm{e}}$ & 66 & 8.04 & 8.27 \\
\hline N II $\lambda 4694.642$ & 23.572 & $+0.100^{\mathrm{d}}$ & 65 & 8.37 & 8.61 \\
\hline N II $\lambda 4774.244$ & 20.646 & -1.257 & 27 & 8.55 & 8.87 \\
\hline N II $\lambda 4779.722$ & 20.646 & -0.587 & 58 & 8.35 & 8.68 \\
\hline N II $\lambda 4781.190$ & 20.654 & -1.308 & 19 & 8.43 & 8.74 \\
\hline N II $\lambda 4793.648$ & 20.654 & -1.095 & 33 & 8.51 & 8.83 \\
\hline $\mathrm{N}_{\text {II }} \lambda 4803.287$ & 20.666 & -0.113 & 88 & 8.23 & 8.57 \\
\hline $\mathrm{N}_{\text {II }} \lambda 4810.299$ & 20.666 & -1.084 & 26 & 8.37 & 8.68 \\
\hline Mean... & $\ldots$ & $\ldots$ & $\ldots$ & $8.29 \pm 0.23$ & $8.59 \pm 0.25$ \\
\hline N III $\lambda 4097.360$ & 27.438 & -0.057 & 184 & 8.23 & 8.39 \\
\hline
\end{tabular}


Table 7

(Continued)

\begin{tabular}{|c|c|c|c|c|c|}
\hline \multirow[t]{2}{*}{ Line } & \multirow{2}{*}{$\begin{array}{c}\chi \\
(\mathrm{eV})\end{array}$} & \multirow[t]{2}{*}{$\log g f$} & \multirow{2}{*}{$\begin{array}{c}W_{\lambda} \\
(\mathrm{m} \AA)\end{array}$} & \multicolumn{2}{|c|}{$\log \epsilon(\mathrm{X})$} \\
\hline & & & & NLTE $^{\mathrm{a}}$ & $\mathrm{LTE}^{\mathrm{b}}$ \\
\hline N III $\lambda 4103.390$ & 27.438 & -0.359 & 169 & 8.44 & 8.56 \\
\hline N III $\lambda 4215.770$ & 36.856 & -0.705 & 18 & 8.41 & 8.54 \\
\hline $\mathrm{N}_{\text {III }} \lambda 4379.201$ & 39.711 & $+1.010^{\mathrm{c}}$ & 133 & 8.31 & 8.90 \\
\hline N III $\lambda 4514.850$ & 35.671 & +0.221 & 112 & $9.15 ?$ & $9.10 ?$ \\
\hline N III $\lambda 4518.140$ & 35.649 & -0.461 & 75 & $9.40 ?$ & $9.33 ?$ \\
\hline N III $\lambda 4523.560$ & 35.657 & -0.353 & 51 & $8.91 ?$ & 8.86 \\
\hline N III $\lambda 4527.860$ & 38.958 & $-0.471^{\mathrm{c}}$ & 23 & 8.41 & 8.88 \\
\hline N III $\lambda 4535.050$ & 38.958 & $-0.170^{\mathrm{c}}$ & 26 & 8.19 & 8.66 \\
\hline N III $\lambda 4539.700$ & 38.645 & -0.452 & 33 & 8.64 & $9.07 ?$ \\
\hline N III $\lambda 4544.840$ & 39.396 & -0.151 & 33 & 8.34 & 8.77 \\
\hline N III $\lambda 4546.330$ & 38.958 & $+0.004^{\mathrm{c}}$ & 21 & 7.90 & 8.35 \\
\hline N III $\lambda 4634.130$ & 30.459 & -0.086 & 115 & 8.63 & 8.47 \\
\hline N III $\lambda 4640.640$ & 30.463 & +0.168 & 131 & 8.50 & 8.37 \\
\hline N III $\lambda 4641.850$ & 30.463 & -0.788 & 139 & $9.53 ?$ & $9.41 ?$ \\
\hline Mean. .. & $\ldots$ & $\ldots$ & $\ldots$ & $8.36 \pm 0.21$ & $8.61 \pm 0.21$ \\
\hline O II $\lambda 4069.623$ & 25.631 & +0.150 & & & \\
\hline O II $\lambda 4069.882$ & 25.638 & +0.344 & 140 & 8.25 & 8.18 \\
\hline О II $\lambda 4072.153$ & 25.650 & +0.552 & 88 & 8.04 & 7.96 \\
\hline O II $\lambda 4085.112$ & 25.650 & -0.189 & 49 & 8.68 & 8.22 \\
\hline O II $\lambda 4092.929$ & 25.665 & -0.308 & 38 & 8.40 & 8.18 \\
\hline O II $\lambda 4366.895$ & 22.999 & -0.348 & 115 & 8.39 & 8.72 \\
\hline O II $\lambda 4414.899$ & 23.442 & +0.172 & 118 & 7.93 & 8.30 \\
\hline O II $\lambda 4416.975$ & 23.419 & -0.077 & 134 & 8.31 & 8.73 \\
\hline O II $\lambda 4452.378$ & 23.442 & -0.788 & 55 & 8.25 & 8.53 \\
\hline O II $\lambda 4590.974$ & 25.661 & +0.350 & 99 & 8.43 & 8.36 \\
\hline O II $\lambda 4595.957$ & 25.661 & -1.033 & & & \\
\hline O II $\lambda 4596.177$ & 25.661 & +0.200 & 87 & 8.38 & 8.30 \\
\hline O II $\lambda 4638.856$ & 22.966 & -0.332 & 110 & 8.34 & 8.67 \\
\hline O II $\lambda 4649.135$ & 22.999 & +0.308 & 151 & 8.08 & 8.52 \\
\hline O II $\lambda 4661.632$ & 22.979 & -0.278 & 90 & 8.09 & 8.39 \\
\hline O II $\lambda 4676.235$ & 22.999 & -0.394 & 67 & 7.95 & 8.23 \\
\hline O II $\lambda 4696.353$ & 28.510 & -1.380 & 16 & 8.07 & 8.36 \\
\hline Mean. .. & $\ldots$ & $\ldots$ & $\ldots$ & $8.24 \pm 0.20$ & $8.38 \pm 0.22$ \\
\hline Ne II $\lambda 4217.169$ & 34.609 & $+0.090^{\mathrm{d}}$ & 45 & 8.59 & 8.57 \\
\hline Ne II $\lambda 4219.745$ & 34.609 & $+0.750^{\mathrm{d}}$ & 99 & 8.60 & 8.64 \\
\hline $\mathrm{Ne}$ II $\lambda 4220.894$ & 34.619 & $-0.060^{\mathrm{d}}$ & & & \\
\hline $\mathrm{Ne}$ II $\lambda 4221.087$ & 34.619 & $-0.740^{\mathrm{d}}$ & 37 & 8.52 & 8.49 \\
\hline $\mathrm{Ne}$ II $\lambda 4224.472$ & 34.632 & $-0.860^{\mathrm{d}}$ & & & \\
\hline $\mathrm{Ne}$ II $\lambda 4224.642$ & 34.632 & $-0.750^{\mathrm{d}}$ & 23 & 8.77 & 8.75 \\
\hline Ne II $\lambda 4231.532$ & 34.619 & $-0.080^{\mathrm{d}}$ & & & \\
\hline $\mathrm{Ne}$ II $\lambda 4231.636$ & 34.619 & $+0.260^{\mathrm{d}}$ & 60 & 8.46 & 8.44 \\
\hline $\mathrm{Ne}$ II $\lambda 4239.911$ & 34.632 & $-0.490^{\mathrm{d}}$ & & & \\
\hline $\mathrm{Ne}$ II $\lambda 4240.105$ & 34.632 & $-0.020^{\mathrm{d}}$ & 50 & 8.62 & 8.59 \\
\hline $\mathrm{Ne}$ II $\lambda 4250.645$ & 34.632 & $+0.150^{\mathrm{d}}$ & 46 & 8.58 & 8.56 \\
\hline $\mathrm{Ne}$ II $\lambda 4397.991$ & 34.814 & $+0.160^{\mathrm{d}}$ & 61 & 8.91 & 8.82 \\
\hline Ne II $\lambda 4430.946$ & 34.749 & $+0.310^{\mathrm{d}}$ & 72 & 8.78 & 8.81 \\
\hline Mean. .. & $\ldots$ & $\ldots$ & 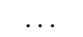 & $8.65 \pm 0.14$ & $8.63 \pm 0.14$ \\
\hline
\end{tabular}

Notes.

a $\left(T_{\text {eff }}, \log g, \xi\right)=(30000,4.10,9.0)$.

b $\left(T_{\text {eff }}, \log g, \xi\right)=(32000,4.20,9.0)$.

c Jeffery (1998).

d Kurucz $g f$-value.

e Wiese et al. (1966).

\section{INTERPRETING THE NEON AND CNO ABUNDANCES}

\subsection{The Context}

Knowledge of the chemical composition of EHe stars has become more complete in recent years. Neon adds a new constraint on proposed origins for these H-deficient stars. In order to exploit this probe fully, the Ne abundance must be considered with the reported abundances of other elements in
Table 8

$\mathrm{He}, \mathrm{C}, \mathrm{N}, \mathrm{O}, \mathrm{Ne}$, and Fe Abundances of the Sample EHe Stars

\begin{tabular}{lccccccc}
\hline \hline \multirow{1}{*}{ Star } & $\left(T_{\text {eff }}, \log g\right)$ & \multicolumn{7}{c}{$\log \epsilon(\mathrm{X})$} \\
\cline { 3 - 8 } & & $\mathrm{He}$ & $\mathrm{C}$ & $\mathrm{N}$ & $\mathrm{O}$ & $\mathrm{Ne}$ & $\mathrm{Fe}^{\mathrm{a}}$ \\
\hline LS IV +6 002 & $(30000,4.10)$ & 11.54 & 9.4 & 8.3 & 8.2 & 8.65 & 7.1 \\
BD-9 4395 & $(24300,2.65)$ & 11.54 & 9.1 & 7.8 & 8.0 & 8.18 & 6.6 \\
LSE 78 & $(18300,2.20)$ & 11.54 & 9.4 & 8.3 & 9.4 & 8.67 & 6.8 \\
BD+10 2179 & $(16375,2.45)$ & 11.54 & 9.3 & 8.1 & 7.9 & 7.87 & 6.2 \\
V1920 Cyg & $(16330,1.80)$ & 11.50 & 9.6 & 8.6 & 9.9 & 8.50 & 6.8 \\
HD 124448 & $(15500,1.90)$ & 11.54 & 9.1 & 8.7 & 8.3 & 7.70 & 7.2 \\
PV Tel & $(13750,1.60)$ & 11.54 & 9.2 & 8.7 & 8.8 & 7.60 & 7.0 \\
\hline
\end{tabular}

Note. a LTE abundance.

EHe stars. A summary of $\mathrm{He}, \mathrm{C}, \mathrm{N}, \mathrm{O}, \mathrm{Ne}$, and $\mathrm{Fe}$ abundances is given in Table 8. NLTE abundances are given for $\mathrm{C}, \mathrm{N}, \mathrm{O}$, and $\mathrm{Ne}$ but not for Fe. For this review, we rely heavily on our earlier analysis (Pandey et al. 2006). Two of our seven stars were not included in the 2006 analysis and two from that analysis are not discussed here. For selected points below, we comment on abundance aspects for other EHes with an abundance analysis.

The following appear to be key points.

Hydrogen: hydrogen is truly a trace element with depletion factors of $10^{3}$ or greater.

Carbon/helium ratio: the $\mathrm{C} / \mathrm{He}$ ratio runs from about $0.3 \%$ to $1.0 \%$ by number for the stars in Table 8 . Five cool EHe stars (Pandey et al. 2001; Pandey \& Reddy 2006) and the two other EHe stars discussed by Pandey et al. (2006) also fall within this range. Two known EHe stars fall well below the range: V652 Her with $\mathrm{C} / \mathrm{He}=0.004 \%$ (Jeffery et al. 1999) and HD 144941 with $\mathrm{C} / \mathrm{He}=0.002 \%$ (Harrison \& Jeffery 1997). One supposes that the scenario accounting for the stars in Table 8 and others with a similar $\mathrm{C} / \mathrm{He}$ ratio will need major revision to account for V652 Her and HD 144941.

Nitrogen: the $\mathrm{N}$ abundance is generally equal to the sum of the initial $\mathrm{C}, \mathrm{N}$, and $\mathrm{O}$ abundances as inferred from an EHe's Fe abundance and standard relations for $\mathrm{C}, \mathrm{N}$, and $\mathrm{O}$ dependences on initial $\mathrm{Fe}$ abundance for normal (i.e., $\mathrm{H}$-normal) dwarfs. This is shown in Figure 9.

Oxygen: oxygen abundances show a large spread: for example, $[\mathrm{O} / \mathrm{H}]$ at $[\mathrm{Fe} / \mathrm{H}] \sim 0$ runs from about +1 to -1 and is, therefore, generally at odds with a simple extrapolation from the $\mathrm{N}$ abundances that $\mathrm{O}$ should be greatly depleted (Figure 10 ). The spread persists to lower $[\mathrm{Fe} / \mathrm{H}]$ with EHe stars with $[\mathrm{O} / \mathrm{H}]$ $\simeq 0$ found at $[\mathrm{Fe} / \mathrm{H}] \simeq-2$.

Neon: our NLTE analysis shows that Ne abundances are approximately independent of a star's Fe abundance (Figure 11). The spread in $\mathrm{Ne}$ abundance at a given $\mathrm{Fe} / \mathrm{H}$ is about 1 dex. Qualitatively, $\mathrm{Ne}$ is similar to $\mathrm{O}$ with respect to spread and $\mathrm{Fe}-$ independence. Neon is not tightly correlated with the $\mathrm{O}$ abundance but the O-richest stars include two of the most Ne-rich and, perhaps significantly, are stars with a strong $s$-process enrichment (V1920 Cyg and LSE 78). This is shown in Figure 12.

$\mathrm{Mg}$ to $\mathrm{Ni}$ : abundances of these metals (relative to $\mathrm{Fe}$ ) follow the relations determined from analyses of Galactic disk and halo stars. In particular, the so-called $\alpha$-elements $(\mathrm{Mg}, \mathrm{Si}, \mathrm{S}, \mathrm{Ca}$, and $\mathrm{Ti})$ in EHe stars fall quite well on the established $[\alpha / \mathrm{Fe}]$ versus $[\mathrm{Fe} / \mathrm{H}]$ trends.

Iron: EHe stars span the metallicity range $[\mathrm{Fe} / \mathrm{H}]=-0.3$ to -2.0 .

$s$-process: several EHe stars appear enriched in $s$-process products. V1920 Cyg and LSE 78, for example, show 50-fold overabundances of the lighter $s$-process products $\mathrm{Y}$ and $\mathrm{Zr}$. 


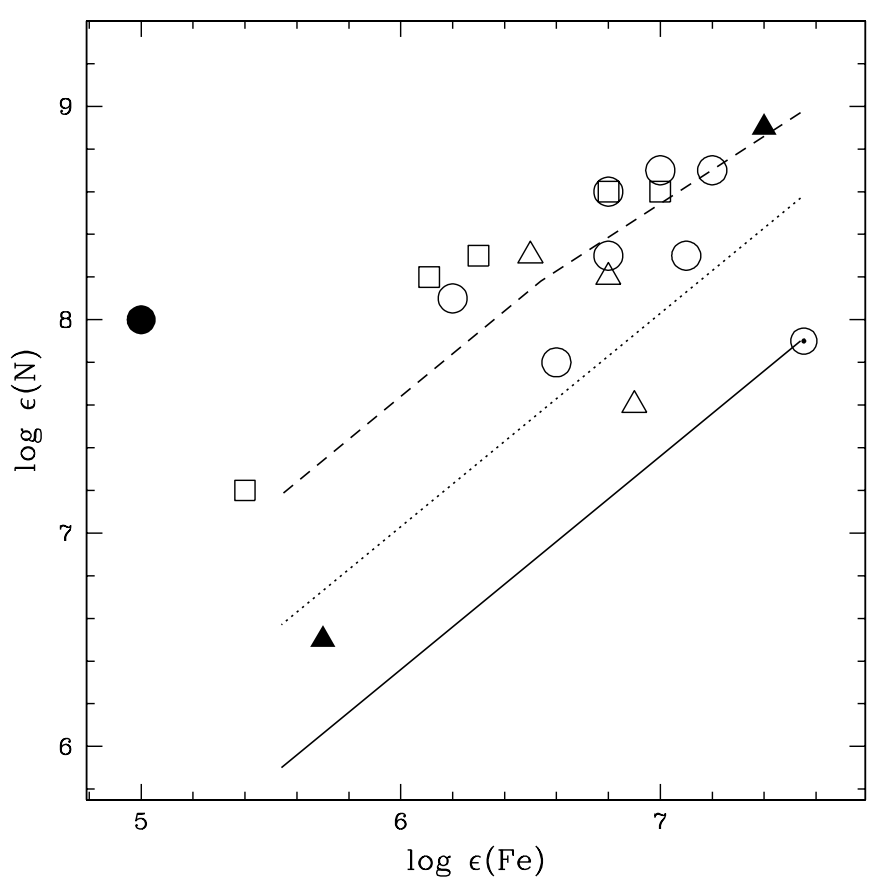

Figure 9. N vs. Fe. Our sample of seven EHes is represented by open circles. Five cool EHes are represented by open squares (Pandey et al. 2001, 2006; Pandey \& Reddy 2006). The results taken from the literature for EHes with C/ He of about 1\% (Drilling et al. 1998; Jeffery et al. 1998) are represented by open triangles. The two EHes of much lower C/He_-V652 Her and HD 144941 - are shown by filled triangles (Jeffery \& Harrison 1997; Harrison \& Jeffery 1997; Jeffery et al. 1999). DY Cen (Jeffery \& Heber 1993) is represented by a filled circle. The circled dot represents the Sun. $\mathrm{N}=\mathrm{Fe}$ is denoted by the solid line. The dotted line represents conversion of the initial sum of $\mathrm{C}$ and $\mathrm{N}$ to $\mathrm{N}$. The dashed line represents the locus of the sum of initial $\mathrm{C}, \mathrm{N}$, and $\mathrm{O}$ converted to $\mathrm{N}$.

Other intriguing abundance anomalies are provided from analyses of RCB and HdC stars which would seem probable relatives of the EHe stars. These anomalies which are undetectable in the EHe stars because the spectroscopic signatures vanish at the higher temperatures include:

${ }^{18} \mathrm{O}$ : a spectacular anomaly is the extraordinarily high ${ }^{18} \mathrm{O}$ abundance seen in cool $\mathrm{HdC}$ and $\mathrm{RCBs}:{ }^{18} \mathrm{O} /{ }^{16} \mathrm{O} \simeq 0.5$ in extreme cases (Clayton et al. 2005, 2007; García-Hernández et al. 2009, 2010). Of course, the $\mathrm{O}$ isotopic abundance ratio requiring detection of the $\mathrm{CO}$ molecule is not measurable for either warm RCBs or the EHes.

Lithium: similarly, measurement of the Li abundance demands a cool atmosphere for detection of the $\mathrm{Li}$ I resonance doublet at $6707 \AA$. Lithium is seen in one of the five $\mathrm{HdC}$ and in four of approximately 30 known RCBs.

Fluorine: a remarkable overabundance of $\mathrm{F}$ was discovered by Pandey (2006) for the cooler EHes and Pandey et al. (2008) for the warmer RCBs. The F I lines vanish at the higher temperatures of the hot EHes discussed here. The F abundances extend to 300 times the solar abundance and the maximum value appears to be independent of a star's Fe abundance. There is a star-to-star spread in $\mathrm{F}$ abundances at a given $[\mathrm{Fe} / \mathrm{H}]$.

Minority RCBs: a few RCBs show highly anomalous [Si/Fe] and $[\mathrm{S} / \mathrm{Fe}]$ ratios. Such stars were called minority RCBs by Lambert \& Rao (1994) with examples including the RCB V $\mathrm{CrA}$ with $[\mathrm{Si} / \mathrm{Fe}] \simeq[\mathrm{S} / \mathrm{Fe}] \simeq+2$ where $\simeq+0.3$ is expected for normal H-rich stars of the same metallicity (Rao \& Lambert 2008). None of the analyzed EHe stars has this minority characteristic but a larger sample may uncover an example. The hot RCB DY Cen, a star known for its reluctance to decline

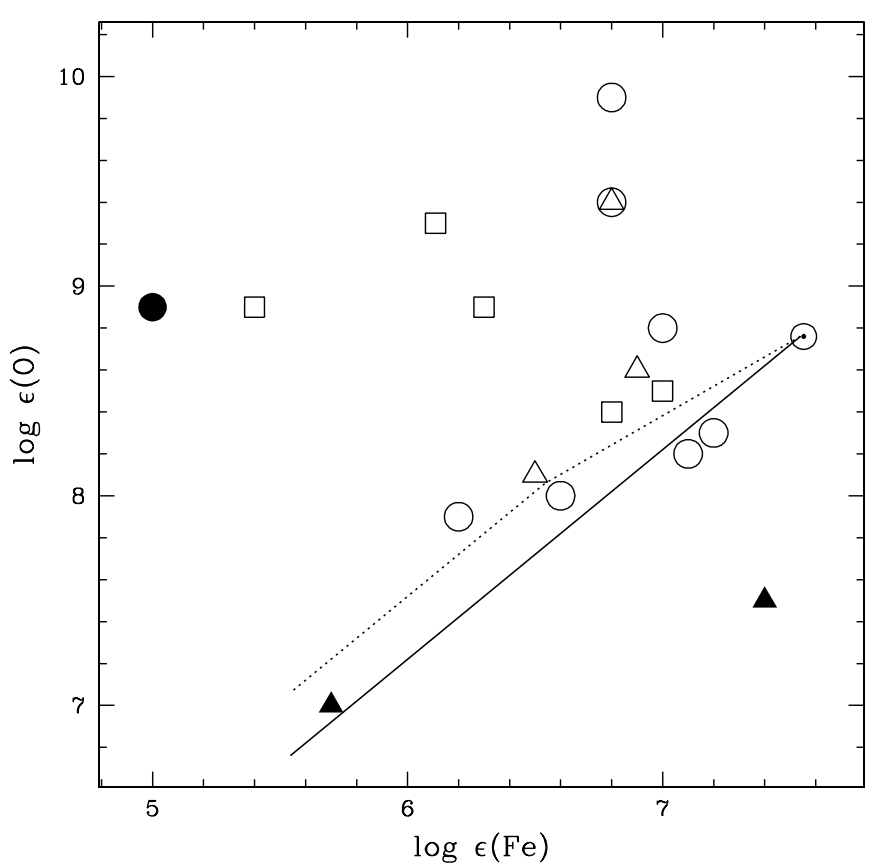

Figure 10. O vs. Fe. Our sample of seven EHes is represented by open circles. Five cool EHes are represented by open squares (Pandey et al. 2001, 2006; Pandey \& Reddy 2006). The results taken from the literature for EHes with C/ He of about 1\% (Drilling et al. 1998; Jeffery et al. 1998) are represented by open triangles. The two EHes of much lower C/He-V652 Her and HD 144941 - are shown by filled triangles (Jeffery \& Harrison 1997; Harrison \& Jeffery 1997; Jeffery et al. 1999). DY Cen (Jeffery \& Heber 1993) is represented by a filled circle. The circled dot represents the Sun. $\mathrm{O}=\mathrm{Fe}$ is denoted by the solid line. The dotted line is from the relation $[\alpha / \mathrm{Fe}]$ vs. $[\mathrm{Fe} / \mathrm{H}]$ for normal disk and halo stars (Ryde \& Lambert 2004).

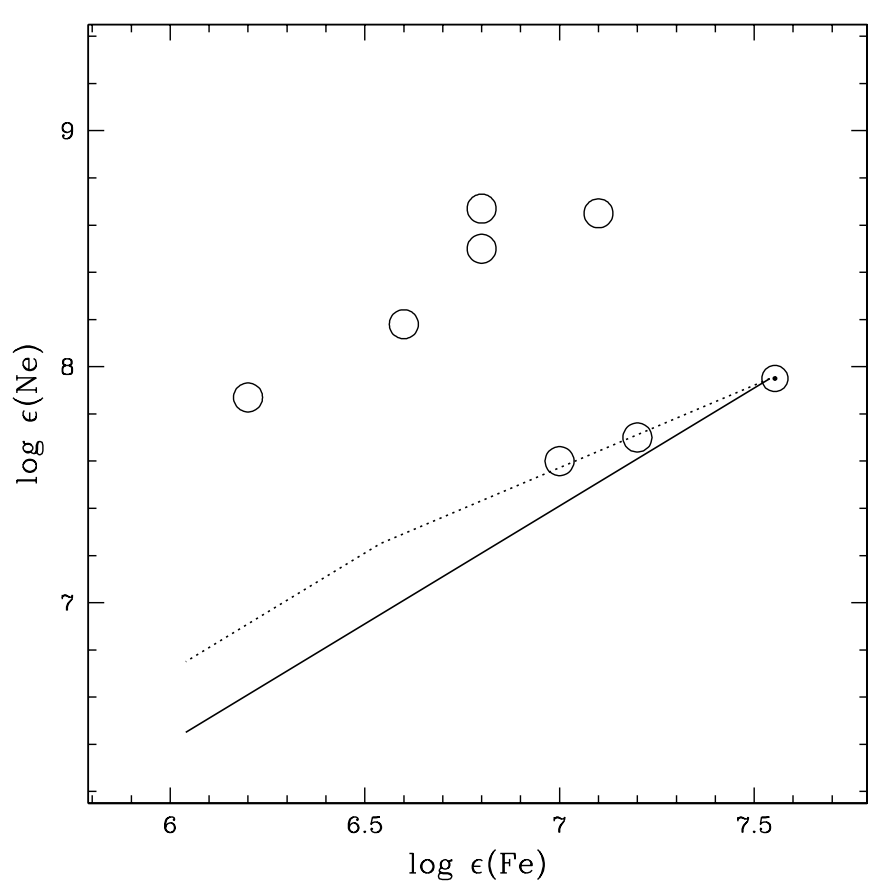

Figure 11. Ne vs. Fe. Our sample of seven EHe stars is represented by circles. The symbol $\odot$ is the Sun. $\mathrm{Ne}=\mathrm{Fe}$ is denoted by the solid line. The dotted line is from the relation $[\alpha / \mathrm{Fe}]$ vs. $[\mathrm{Fe} / \mathrm{H}]$ for normal disk and halo stars (Ryde \& Lambert 2004).

from maximum light, is a minority star and might almost be called an EHe star. 


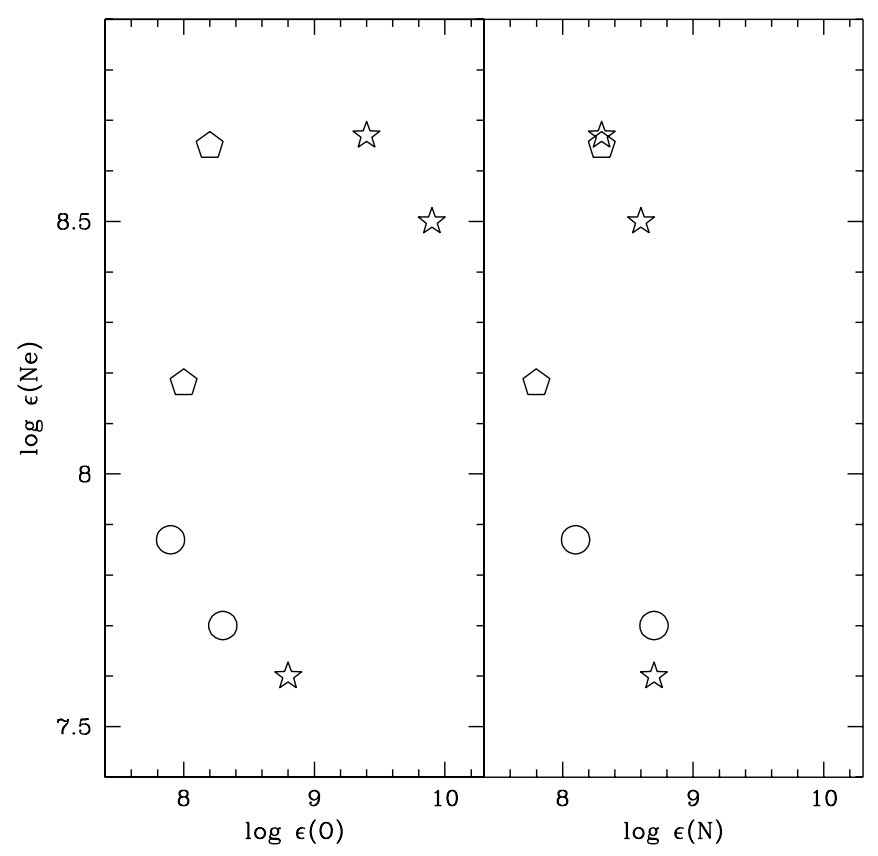

Figure 12. Left: $\mathrm{Ne}$ vs. O. Our sample of seven EHe stars. s-processenriched EHes are represented by stars. EHes with no $s$-process enrichment are represented by circles. EHes with no information on $s$-process enrichment are represented by pentagons. Right: Ne vs. N. The symbols have the same meaning as in the left panel.

\subsection{The Double-degenerate Scenario}

\subsubsection{A Recipe}

In the simplest implementation of the DD scenario, an $\mathrm{He}$ WD merges with a $\mathrm{C}-\mathrm{O}$ WD to produce an EHe star without nucleosynthesis occurring during the merger, an occurrence which we term a cold merger. Subsequent evolution of the $\mathrm{H}$-deficient supergiant star is assumed not to result in further changes of surface composition. Under these assumptions, it is possible to predict the composition of the EHe star created by the merger. The merger seems certain to result in a rapidly rotating compact object. Expansion of the envelope to produce the EHe giant will through conservation of angular momentum result in a less rapidly rotating star. Here, one recalls that slowly rotating normal giants evolve from parts of the main sequence where rapidly rotating stars are common. Line profiles of the EHes indicate a high macroturbulent velocity to which the projected rotational velocity may be a significant contributor, e.g., BD-9 ${ }^{\circ} 4395$ 's line profiles suggest that the projected rotational velocity is about $40 \mathrm{~km} \mathrm{~s}^{-1}$.

In this picture, the merger involves mixing without nuclear cooking of two principal ingredients: the He WD and the former He shell of the $\mathrm{C}-\mathrm{O}$ WD. ${ }^{5}$ A third potential ingredient may be provided by those layers of the $\mathrm{C}-\mathrm{O}$ WD immediately below its He shell which may be disturbed and mixed with the accreted He WD during the merger. One or both WDs before the merger may contain a thin outer layer of $\mathrm{H}$-rich material but since $\mathrm{H}$ is very deficient in the merged star, we may neglect these H-rich layers in the following discussion of abundances of major elements resulting from a cold merger. Gravitational settling may occur in both the He and the $\mathrm{C}-\mathrm{O}$ WDs ahead of the merger. Effects of settling in the He WD will be erased when the star is merged with the $\mathrm{C}-\mathrm{O}$ WD. The merger event presumably stirs up the

\footnotetext{
5 Here, the He WD has an He-core and is to be distinguished from a DB WD, a star with an $\mathrm{He}$ atmosphere but a $\mathrm{C}-\mathrm{O}$ core.
}

C-O WD's He shell and again the effects of gravitational settling are negated. Our recipe assumes that the He WD is thoroughly mixed with the thin $\mathrm{He}$ shell of the $\mathrm{C}-\mathrm{O}$ WD. Implications of mergers involving $\mathrm{H}$-rich layers are explored briefly in an attempt to account for the Li-rich RCB stars. To predict the merged star's composition we need the masses and compositions of the ingredients.

\subsubsection{One Ingredient-The He WD}

In principle, an $\mathrm{He}$ WD is created from low mass main sequence stars but this requires a time exceeding the age of the Galaxy. Thus, the He WD in a DD scenario must be a product of a binary system experiencing mass loss and probably mass transfer. Iben et al. (1997) predict the mass distribution of $\mathrm{He}$ and $\mathrm{C}-\mathrm{O}$ WDs expected to result from evolution of close binaries: $M(\mathrm{He}) \simeq 0.3 \pm 0.1 M_{\odot}$ and $M(\mathrm{C}-\mathrm{O}) \simeq 0.6 \pm 0.1 M_{\odot}$. Such an He WD's composition is dominated by He and N: the mass fraction $\mu(\mathrm{He})$ of $\mathrm{He}$ is essentially unity and, thanks to $\mathrm{H}$ burning by the $\mathrm{CNO}$ cycles, the $\mathrm{N}$ abundance is the sum of the initial $\mathrm{C}, \mathrm{N}$, and $\mathrm{O}$ abundances, say $\mu(\mathrm{CNO})_{0}$ where 0 denotes that the initial $\mathrm{C}, \mathrm{N}$, and $\mathrm{O}$ abundances will be dependent on the initial metallicity (here inferred from the Fe abundance). Mass fractions of $\mathrm{C}$ and $\mathrm{O}$ in the He WD may be taken to be zero. Heavier elements will have their initial mass fractions. Thus, the masses of helium and nitrogen contributed to the merged star assuming a conservative cold merger are essentially $M(\mathrm{He})$ and $\mu(\mathrm{CNO})_{0} M(\mathrm{He})$, respectively.

\subsubsection{Another Ingredient-The He Shell of the $C-O W D$}

For the He shell of the $\mathrm{C}-\mathrm{O}$ WD, estimates of the mass and composition of the $\mathrm{He}$ shell should be obtained from calculations of binary star evolution that result in appropriate $\mathrm{He}$ and $\mathrm{C}-\mathrm{O}$ WD pairs, but understandably such calculations appear not to have been reported. Therefore, we take estimates from calculations for the inner regions of single stars in their AGB phase prior to loss of their H-rich envelopes. In such cases, the mass of the He shell is approximately $0.02 M_{\odot}$ for $1-3 M_{\odot}$ stars but decreases to $0.002 M_{\odot}$ for the more massive stars. We denote this mass by $M(\mathrm{C}-\mathrm{O}: \mathrm{He})$.

Early calculations showed that the He shell was primarily comprised of ${ }^{4} \mathrm{He}$ and ${ }^{12} \mathrm{C}$ with mass fractions of about 0.75 and 0.20 , respectively, with ${ }^{16} \mathrm{O}$ having a mass fraction of only about 0.01 (Schönberner 1979). We make use of calculations by Karakas (2010) (and A. I. Karakas 2010, private communication) for stars with masses of 1-6 $M_{\odot}$ and with initial compositions $Z=0.0001-0.02$. Adopted compositions are the average of the He shell's composition just prior to third dredge-up and at the point at which the star leaves the AGB. Mass fractions of ${ }^{4} \mathrm{He}$, ${ }^{12} \mathrm{C}$, and ${ }^{16} \mathrm{O}$ are consistent with Schönberner's estimates. In Karakas et al.'s calculations (A. I. Karakas 2010, private communication, and others like Schönberner 1979), the He mass fraction $\mu(\mathrm{He})_{\mathrm{C}-\mathrm{O}: \mathrm{He}}$ is about 0.75 , almost independent of mass and composition. The ${ }^{12} \mathrm{C}$ mass fraction $\mu(\mathrm{C})_{\mathrm{C}-\mathrm{O}: \mathrm{He}} \simeq 0.20$, again with little dependence on mass and composition. ${ }^{14} \mathrm{~N}$ is effectively cleansed from the region and we take its mass fraction to be zero. The ${ }^{16} \mathrm{O}$ mass fraction is $\mu(\mathrm{O})_{\mathrm{C}-\mathrm{O}: \mathrm{He}} \simeq 0.005$.

Of particular interest to attempts to match the EHe compositions is that the He shell has enhanced ${ }^{22} \mathrm{Ne}$ and ${ }^{19} \mathrm{~F}$ abundances. Mass fractions of these two nuclides are dependent on the mass of the initial star but are not particularly sensitive to the initial metal mass fraction $Z$. The ${ }^{22} \mathrm{Ne}$ is synthesized from ${ }^{14} \mathrm{~N}$ by $\alpha$-capture, first to ${ }^{18} \mathrm{O}$ and then to ${ }^{22} \mathrm{Ne}$ : its abundance peaks in stars of about $3 M_{\odot}$ reaching a mass fraction of about 0.05 , 
a value not greatly dependent on the initial metallicity of the star. The ${ }^{22} \mathrm{Ne}$ mass fraction decreases to lower initial stellar masses by a factor that is metallicity dependent: at $Z=0.008$, the mass fraction for a $1 M_{\odot}$ star is a factor of six below that for a $3 M_{\odot}$ star. At higher masses than $3 M_{\odot},{ }^{22} \mathrm{Ne}$ is destroyed by $\alpha$-particles and converted to ${ }^{25} \mathrm{Mg}$ and ${ }^{26} \mathrm{Mg}$.

Synthesis of ${ }^{19} \mathrm{~F}$ occurs from ${ }^{15} \mathrm{~N}$ by ${ }^{15} \mathrm{~N}(\alpha, \gamma){ }^{19} \mathrm{~F}$ in competition with $\left.{ }^{19} \mathrm{~F}(\alpha, p)\right)^{22} \mathrm{Ne}$ with ${ }^{15} \mathrm{~N}$ produced by either ${ }^{14} \mathrm{~N}(n$, $p){ }^{14} \mathrm{C}(\alpha, \gamma){ }^{18} \mathrm{O}(p, \alpha){ }^{15} \mathrm{~N}$ or ${ }^{14} \mathrm{~N}(\alpha, \gamma){ }^{18} \mathrm{~F}\left(\beta^{+}\right){ }^{18} \mathrm{O}(p, \alpha){ }^{15} \mathrm{~N}$ with neutrons from ${ }^{13} \mathrm{C}(\alpha, n){ }^{16} \mathrm{O}$ and protons from ${ }^{14} \mathrm{~N}(n, p){ }^{14} \mathrm{C}$. The ${ }^{19} \mathrm{~F}$ mass fraction is a maximum for $M \simeq 3 M_{\odot}$ decreasing by about factors of 20-30 for $1 M_{\odot}$ and 10 for $6 M_{\odot}$. At maximum, the mass fraction $\mu(\mathrm{F})_{\mathrm{C}-\mathrm{O}: \mathrm{He}} \simeq 1 \times 10^{-4}$.

Other series of calculations for AGB stars introduce convective overshoot at the base of the He-burning thermal pulse in the He shell. ${ }^{6}$ Overshoot necessarily brings more ${ }^{16} \mathrm{O}$ (and ${ }^{12} \mathrm{C}$ ) into the shell from the top of the $\mathrm{C}-\mathrm{O}$ core. Various implementations of convective overshoot have been reported in the literature: for example, Herwig (2000) (see also Herwig 2006) reports for a star of initial mass $3 M_{\odot}$ that the He shell at the last thermal pulse has mass fractions of 0.41 and 0.18 for ${ }^{12} \mathrm{C}$ and ${ }^{16} \mathrm{O}$, respectively, showing an order of magnitude increase in the ${ }^{16} \mathrm{O}$ mass fraction from the calculation without this convective overshoot. One may anticipate the possibility that $\mathrm{O}$ abundances in EHe stars may offer an indirect test of calculations with and without convective overshoot extending into the $\mathrm{C}-\mathrm{O}$ core.

\subsubsection{Mixing the Ingredients}

With estimates of the compositions of the two principal ingredients, we may predict the outcomes of a cold merger. As noted above, we may set $\mathrm{H}$ aside because its abundance in the EHe star can be readily accounted for by adding a small $\mathrm{H}$-rich skin to the $\mathrm{He} \mathrm{WD}$ and/or the $\mathrm{C}-\mathrm{O}$ WD. Estimates for other elements are as follows.

The $\mathrm{C} / \mathrm{He}$ ratio: helium is provided overwhelmingly by the $\mathrm{He} \mathrm{WD}$ and $\mathrm{C}$ exclusively by the $\mathrm{C}-\mathrm{O}$ WD's He shell. The predicted $\mathrm{C} / \mathrm{He}$ ratio by number is

$$
\frac{\mathrm{C}}{\mathrm{He}} \simeq \frac{A(\mathrm{He})}{A(\mathrm{C})} \frac{\mu(\mathrm{C})_{\mathrm{C}-\mathrm{O}: \mathrm{He}} M(\mathrm{C}-\mathrm{O}: \mathrm{He})}{M(\mathrm{He})},
$$

where $A(\mathrm{X})$ denotes the atomic weight of $\mathrm{X}$.

With $\mu(\mathrm{C})_{(\mathrm{C}-\mathrm{O}: \mathrm{He})} \simeq 0.2, M(\mathrm{C}-\mathrm{O}: \mathrm{He}) \simeq 0.02 M_{\odot}$, and $M(\mathrm{He}) \simeq 0.3 \mathrm{M}_{\odot}, \mathrm{C} / \mathrm{He} \simeq 0.4 \%$. Since the observed range of the $\mathrm{C} / \mathrm{He}$ ratio is from $0.3 \%$ to $1.0 \%$, our prediction accounts well for the lower end of the observed range. It is possible to account for the upper end with not implausibly different choices for the three variables. If additional ${ }^{12} \mathrm{C}$ is needed, it may be provided by the third ingredient, the "surface" layers of the $\mathrm{C}-\mathrm{O}$ WD where the ${ }^{12} \mathrm{C}$ mass fraction may approach $0.5-0.8$. For example, an additional contribution of $0.01 M_{\odot}$ with a ${ }^{12} \mathrm{C}$ mass fraction of 0.5 raises the $\mathrm{C} / \mathrm{He}$ to $1 \%$ after the merger.

Although not present in our sample of EHes for which we have derived the $\mathrm{Ne}$ abundance, two EHes-V652 Her and HD 144941 - as noted above have lower $\mathrm{C} / \mathrm{He}$ ratios by two orders of magnitude: $\mathrm{C} / \mathrm{He} \simeq 0.003 \%$. An interpretation is that these stars result from a DD scenario involving a pair of $\mathrm{He}$ WDs. An alternative possibility based on the above equation

\footnotetext{
6 This episode of convective overshoot is to be distinguished from convective overshoot at the base of the $\mathrm{H}$-rich convective envelope into the top of the $\mathrm{He}$ shell of an AGB star. This affects operation of the $s$-process in the He shell between thermal pulses and also the composition of the surface layers - see Karakas et al. (2010) for a discussion.
}

is that the $\mathrm{C}-\mathrm{O}$ WD in the merger had an unusually small $\mathrm{He}$ shell around the $\mathrm{C}-\mathrm{O}$ WD, as might be anticipated for a star of intermediate mass.

The $\mathrm{N}$ abundance: nitrogen, a product of CNO-cycling, is contributed by the $\mathrm{He}$ WD which also is the dominant contributor of mass to the merger and, hence, the leading supplier of a reference element such as Fe. These circumstances explain quite naturally why the observed $\mathrm{N}$ abundance in stars of different $\mathrm{Fe}$ abundance is equal to the sum of the initial $\mathrm{C}, \mathrm{N}$, and $\mathrm{O}$ abundances.

The oxygen abundance: the $\mathrm{O}$ abundance is given by

$$
\frac{\mathrm{O}}{\mathrm{He}} \simeq \frac{A(\mathrm{He})}{A(\mathrm{O})} \frac{\mu(\mathrm{O})_{\mathrm{C}-\mathrm{O}: \mathrm{He}} M(\mathrm{C}-\mathrm{O}: \mathrm{He})}{M(\mathrm{He})},
$$

with $\mu(\mathrm{O})_{(\mathrm{C}-\mathrm{O}: \mathrm{He})} \simeq 0.005, M(\mathrm{C}-\mathrm{O}: \mathrm{He}) \simeq 0.02 M_{\odot}$, and $M(\mathrm{He}) \simeq 0.3 \mathrm{M}_{\odot}, \mathrm{O} / \mathrm{He} \simeq 0.008 \%$ corresponding to an abundance $\log \epsilon(\mathrm{O}) \simeq 7.5$, a value at the lower bound of the observed abundances.

There appear to be two possibilities by which to increase the predicted $\mathrm{O}$ abundances: (1) add the third ingredient, i.e., surface layers from the $\mathrm{C}-\mathrm{O} \mathrm{WD}$, when, for example, a mass of $0.01 M_{\odot}$ and an ${ }^{16} \mathrm{O}$ mass fraction of 0.5 raises the $\mathrm{O}$ abundance to 9.2 , the maximum observed value; (2) increase the $\mathrm{O}$ mass fraction in the He shell to 0.02 , a value expected if convective overshoot during the AGB phase extends into the $\mathrm{C}-\mathrm{O}$ core, and then the predicted $\mathrm{O}$ abundance is 8.1. Both of these possibilities may depend on individual properties of the stars involved in the merger and, thus, might account for the observed spread in $\mathrm{O}$ abundances among $\mathrm{EHe}$ (and RCB) stars. As long as the responsible agent is not metallicity dependent, the lack of a trend of $\mathrm{O}$ abundance with metallicity is accounted for.

The Neon abundance: the Ne abundance is

$$
\frac{\mathrm{Ne}}{\mathrm{He}} \simeq \frac{A(\mathrm{He})}{A\left({ }^{22} \mathrm{Ne}\right)} \frac{\mu(\mathrm{Ne})_{\mathrm{C}-\mathrm{O}: \mathrm{He}} M(\mathrm{C}-\mathrm{O}: \mathrm{He})+\mu(\mathrm{Ne})_{0} M(\mathrm{He})}{M(\mathrm{He})},
$$

where contributions from the He shell of the $\mathrm{C}-\mathrm{O}$ WD and the original Ne content of the He WD are included.

With $\mu(\mathrm{Ne})_{(\mathrm{C}-\mathrm{O}: \mathrm{He})} \simeq 0.05, M(\mathrm{C}-\mathrm{O}: \mathrm{He}) \simeq 0.02 M_{\odot}$, and $M(\mathrm{He}) \simeq 0.3 \mathrm{M}_{\odot}, \mathrm{Ne} / \mathrm{He} \simeq 0.06 \%$ corresponding to an abundance $\log \epsilon(\mathrm{Ne}) \simeq 8.4$, a value equal to the upper bound of the observed abundances (Table 8 and Figure 11). Lower $\mathrm{Ne}$ abundances are readily achieved because the Ne mass fraction is lower in all but $3 M_{\odot}$ stars. Karakas's predictions (Karakas 2010 and A. I. Karakas 2010, private communication) encompass an order of magnitude range in Ne mass fractions and the observed $\mathrm{Ne}$ abundances from our (small) sample of EHe stars show a factor of five spread. In all cases but two (HD 124448 and PV Tel), the Ne abundance is appreciably greater than the presumed initial abundance based on the Fe abundance and likely relation between initial $\mathrm{Ne}$ and $\mathrm{Fe}$ abundances.

The fluorine abundance: fluorine is not detectable in these hot EHes but its abundance is known for cooler EHes and the warmer RCBs, as noted above. The maximum mass fraction for $\mathrm{F}$ in the He shell gives an abundance $\log \epsilon(\mathrm{F}) \simeq 5.7$ but observed abundances are about 1 dex higher. This may suggest that the nucleosynthesis of $\mathrm{F}$ has been underestimated in the He shell, or the derived $\mathrm{F}$ abundance for cooler EHes has been overestimated. This gap might also be bridged by NLTE calculations of F I line formation; such calculations for $\mathrm{Ne}$ I, an atom with a not dissimilar atomic structure, show an almost 1 dex reduction of the observed LTE abundances.

The ${ }^{18} \mathrm{O}$ abundance: extraordinary amounts of ${ }^{18} \mathrm{O}$ are present with the ${ }^{18} \mathrm{O}$ abundance exceeding that of ${ }^{16} \mathrm{O}$ in several stars. 
The ${ }^{18} \mathrm{O} /{ }^{16} \mathrm{O}$ ratio is higher and the ${ }^{18} \mathrm{O}$ abundance is lower in most of the RCBs where CO lines are detectable; GarcíaHernández et al. (2010) speculate that a late dredge-up in an $\mathrm{HdC}$ star is responsible for these differences between $\mathrm{HdC}$ and RCB stars. These high ${ }^{18} \mathrm{O}$ abundances cannot be explained by the two or even the three ingredient recipe describing the DD scenario as a cold merger. One may wonder if the $\mathrm{C}-\mathrm{O}$ WD's He shell has a composition that is not a complete replica of an $\mathrm{He}$ shell of a single star, the model we have adopted for these calculations. Perhaps the synthesis of ${ }^{22} \mathrm{Ne}$ from ${ }^{14} \mathrm{~N}$ is incomplete and some ${ }^{18} \mathrm{O}$ remains. But one wonders if this suspicion may be reconciled with the fact that the maximum ${ }^{18} \mathrm{O}$ abundances for the $\mathrm{HdC}$ stars $\left(\log \epsilon\left({ }^{18} \mathrm{O}\right) \simeq 8.6\right)$ are greater than the $\mathrm{Ne}$ abundances $(\log \epsilon(\mathrm{Ne}) \simeq 8.5)$ in the EHe stars. Is such fine tuning possible?

Clayton et al. (2005) considered ${ }^{18} \mathrm{O}$ synthesis to occur by nuclear processing during accretion of $\mathrm{He}$ WD material by the $\mathrm{C}-\mathrm{O}$ WD. In the processing, ${ }^{14} \mathrm{~N}$ is converted to ${ }^{18} \mathrm{O}$ by $\alpha$-capture. The observed ${ }^{18} \mathrm{O}$ abundances are not sufficiently great to have sensibly reduced the ${ }^{14} \mathrm{~N}$ abundances: the ${ }^{18} \mathrm{O}$ abundances are factors of four to 20 less than the ${ }^{14} \mathrm{~N}$ abundances. However, fine tuning is required in order not to deplete entirely the ${ }^{14} \mathrm{~N}$ supply and also to prevent conversion of significant amounts of ${ }^{18} \mathrm{O}$ by $\alpha$-capture to ${ }^{22} \mathrm{Ne}$.

The $s$-process: enrichment of $s$-process nuclides likely in the $\mathrm{C}-\mathrm{O}$ WD's He shell will be diluted by the absence of enrichment in the He WD. Thus, the enrichment will be diluted by about an order or magnitude if $M(\mathrm{He}) \simeq 0.3 M_{\odot}$ and $M(\mathrm{He})_{\mathrm{C}-\mathrm{O}} \simeq 0.02 M_{\odot}$. Two of the EHes are observed enriched in $\mathrm{Y}$ and $\mathrm{Zr} 50$-fold and another 10-fold. Other stars have lower enrichment levels. Although a 100- to 500-fold enrichment is within expected levels for isolated AGB stars, the general lack of a large $s$-process enrichment of these EHes, as well as the cool EHes (Pandey et al. 2001) and the warmer RCBs (Asplund et al. 2000) implies that the He shell of the C-O WD was not itself greatly $s$-process enriched. This would seem to confirm suspicions that the He shells participating in the DD scenario may not be near-copies of He shells of isolated AGB stars.

Lithium: lithium is, of course, not observable in EHe stars but its presence in several RCBs and in one of five known HdCs suggests that it is probably present in at least some EHe stars. Therefore, the challenge exists to account for the Li abundance in the DD scenario.

An initial supposition is that the $\mathrm{Li}$ was present in the H-rich skin around the WDs. Then, the observed $\mathrm{Li} / \mathrm{H}$ ratio for the $\mathrm{RCB}$ star is the mass-weighted mean of the $\mathrm{Li} / \mathrm{H}$ ratio in the two H-rich skins. For the four RCBs with $\mathrm{Li}, \log \mathrm{Li} / \mathrm{H}$ ranges from -1.7 for RZ Nor to -4.8 for SU Tau (Asplund et al. 2000) or Li abundances of 10.3 to 7.2 on the usual logarithmic scale where the $\mathrm{H}$ abundance is 12.0 . Such extraordinarily high Li abundances are observed nowhere else: for example, the Li-rich carbon stars have Li abundances only (!) in the range from three to five (Abia et al. 1999).

In the usual scheme of $\mathrm{Li}$ synthesis known as the Cameron-Fowler mechanism (Cameron \& Fowler 1971), Li as ${ }^{7} \mathrm{Li}$ is synthesized from ${ }^{3} \mathrm{He}$ by the chain ${ }^{3} \mathrm{He}\left({ }^{4} \mathrm{He}, \gamma\right){ }^{7} \mathrm{Be}\left(e^{-}, v\right)$ ${ }^{7} \mathrm{Li}$. The potential reservoir of ${ }^{3} \mathrm{He}$ is the star's original supply of ${ }^{3} \mathrm{He}$ and ${ }^{2} \mathrm{H}$ (which is burnt to ${ }^{3} \mathrm{He}$ in pre-main sequence phase) and additional ${ }^{3} \mathrm{He}$ provided by operation of the $p p$-chains in low mass main sequence stars. The original ${ }^{3} \mathrm{He} / \mathrm{H}$ after ${ }^{2} \mathrm{H}$ burning will have been about $2 \times 10^{-5}$. In low mass stars, a layer of enriched ${ }^{3} \mathrm{He}$ exists outside the H-burning core. The abundance can reach $10^{-3}$ of that of $\mathrm{H}$ over a shell about $0.2 M_{\odot}$ in thickness (Iben 1967). Protons are not directly involved in the Cameron-Fowler mechanism but the temperature of the synthesis site may be influenced by the mass exterior to the site.

In the case of the Li-rich normal carbon stars, the site is the high-temperature base of the H-rich convective envelope in an intermediate-mass AGB star. In this environment, the observed range of Li abundances can be achieved. To achieve a higher abundance (i.e., a higher $\mathrm{Li} / \mathrm{H}$ ratio), it seems necessary to reduce the mass of the $\mathrm{H}$ into which products $\left({ }^{7} \mathrm{Be}\right.$ and then ${ }^{7} \mathrm{Li}$ ) of ${ }^{3} \mathrm{He}$ consumption are mixed. Efficiency of ${ }^{7} \mathrm{Li}$ production might be maximized were the ${ }^{3} \mathrm{He}$ consumed in a layer completely devoid of $\mathrm{H}$; this would remove the loss of ${ }^{7} \mathrm{Be}$ and ${ }^{7} \mathrm{Li}$ by proton capture but these nuclides would still be prone to destruction by $\alpha$-capture. Further exploration of ${ }^{7} \mathrm{Li}$ synthesis will require very detailed calculations of nucleosynthesis in close binary systems.

\subsection{The Final-flash Scenario}

Several classes of H-deficient hot luminous post-AGB stars are believed to have resulted from an FF scenario. Compositions of these stars offer a direct point of comparison for the EHe (also $\mathrm{RCB}$ and HdC) stars and, therefore, a test of the FF scenario as an origin for some EHe stars.

A valuable review of the observed compositions and theoretical origins of hot H-deficient post-AGB stars was provided by Werner \& Herwig (2006). Their Table 1 clearly shows that the several families ([WCL], [WCE], [WC]-PG1159, and PG1159) of such post-AGB stars have compositions differing in several distinctive ways from the compositions of EHe and RCB stars. In particular, the relative $\mathrm{C} / \mathrm{He}$ ratio is quite different. The $(\mathrm{He}, \mathrm{C})$ mass fractions are roughly in the range $(0.30,0.60)$ to $(0.85,0.15)$ whereas the EHes are close to $(0.98,0.02)$ (Werner et al. 2008). This contrast is, of course, very largely attributable to the $\mathrm{He}$ contribution by the He WD to the DD scenario. Several of the post-AGB stars show a residue of their original $\mathrm{H}$ with a mass fraction of as much as 0.35 , but a lower value is more common. (These are hot stars and, therefore, detection of $\mathrm{H}$ lines is difficult.) In other respects, the compositions of the post-AGB and EHe stars are more similar: the $\mathrm{O}$ mass fractions show a starto-star spread but the highest value for a post-AGB star $(0.20$, Werner et al. 2008) is several times higher than the maximum value for an $\mathrm{EHe}$. The $\mathrm{F}$ and $\mathrm{Ne}$ abundances are similar for the two groups of stars.

Theoretical FF models are discussed by Werner \& Herwig (2006). These models differ as to when the He-shell flash (thermal pulse) occurs that restores the star to the post-AGB track as an EHe-like star. If the thermal pulse occurs when the star is on the AGB, it is termed an AFTP and may account for the relatively $\mathrm{H}$-rich stars known as hybrid-PG1159 stars. If the thermal pulse occurs in the post-AGB period of approximately constant luminosity, it is an LTP ( $\mathrm{L}=$ Late) and is predicted to create an $\mathrm{H}$-deficient star with some $\mathrm{H}$, say a mass fraction of about 0.02. FG Sge may have experienced an LTP_recently! Finally, if the thermal pulse is delayed until the star is on the WD cooling track, it is a VLTP (V = very). Sakurai's object is considered to have undergone a VLTP_-very recently! Although these post-AGB FF scenarios fail to account for the EHes, one may note some similarities with the ideas behind the DD scenario. In particular, convective extramixing into the "surface" layers of the $\mathrm{C}-\mathrm{O}$ core is invoked in both cases to account for the spread in the $\mathrm{O}$ abundances, and high $\mathrm{F}$ and $\mathrm{Ne}$ abundances in the $\mathrm{He}$ shell around the $\mathrm{C}-\mathrm{O}$ core of the AGB star to explain the $\mathrm{F}$ and $\mathrm{Ne}$ overabundances in both kinds of $\mathrm{H}$-deficient stars. 


\section{CONCLUDING REMARKS}

Understanding the origins of peculiar stars often awaits recognition of predecessors and descendants along the evolutionary sequence. The sequence may provide clues not always provided by even detailed observational studies of a single class of peculiar star in the sequence. Even more important than expanded observational studies is the development of theoretical understanding of relevant stellar models and associated nucleosynthesis. The EHe stars well illustrate these remarks.

The first EHe HD 124448 was discovered by Popper (1942) at the McDonald Observatory and this was followed ten years later by discovery of PV Tel, alias HD 168476, by Thackeray \& Wesselink (1952) at the Radcliffe Observatory. Today, the register of EHes totals about 30 (Jeffery 1996). ${ }^{7}$ Today, the chemical compositions of EHe stars are quite well determined with our estimates of $\mathrm{Ne}$ abundances adding one more data point. Similarities in composition argue for an evolutionary link between EHe and RCB stars and less convincingly between these stars and the HdC stars of which only five are known and their spectra blessed with a rich array of molecular lines render accurate abundance analysis difficult. Theoretical ideas have centered on two possibilities: the DD and the FF scenarios.

While the FF scenario may account for H-deficient stars like FG Sge and Sakurai's object (as noted above), the argument eliminating it as the origin of EHe stars is a fusion of two principal points. First, the compositions of H-deficient central stars of planetary nebulae differ in critical aspects from those of EHe stars. As noted in Section 5.3, the He and C mass fractions of the central stars differ greatly from these quantities as found for EHe (and RCB) stars. Second, the observed compositions of the central stars are reasonably well accounted for by models of final He shell flashes in post-AGB stars. Thus, a safe conclusion would appear to be that the FF scenario cannot account for the EHe stars.

Identification of the EHe (and RCB) stars with the DD scenario depends on the correspondence between the measured chemical compositions and semi-quantitative theoretical estimates resulting from the merger of an $\mathrm{He}$ WD with a $\mathrm{C}-\mathrm{O}$ WD. Predictions for a cold merger seem especially sensitive to the adopted composition of the He shell around the $\mathrm{C}-\mathrm{O}$ WD before the merger and the extent to which mixing during the merger may incorporate material from the surface layers of the $\mathrm{C}-\mathrm{O}$ WD. An additional uncertainty concerns the extent of nucleosynthesis occurring during the merger; the proposal that the ${ }^{18} \mathrm{O}$ seen in cool HdC and RCB stars is synthesized from ${ }^{14} \mathrm{~N}$ during the merger was noted (Clayton et al. 2007).

Although work remains for quantitative spectroscopists to do, we close with the thought that the larger challenges remain in the area of theoretical modeling of single stars in order to refine prediction for the several forms of the FF scenario and of double stars that through common envelope stages and mass loss provide the necessary stage for the DD scenario of a close binary of an $\mathrm{He}$ and a $\mathrm{C}-\mathrm{O}$ WD that merge with possibly a concluding episode of nucleosynthesis to provide an $\mathrm{EHe}, \mathrm{RCB}$, or an $\mathrm{HdC}$.

We thank the referee for a constructive report. We are indebted to Amanda Karakas for providing details of her calculations of AGB stars. We thank Katia Cunha for providing her Ne I line list for HD 35299. G.P. thanks Baba Verghese for the help provided in installing the TLUSTY/SYNSPEC codes. G.P. is thankful

\footnotetext{
7 H-deficient binaries such as KS Per and $v$ Sgr are an unrelated and even rarer type of stars.
}

to Carlos Allende Prieto for his useful suggestions in making the TLUSTY models. We thank Ivan Hubeny for testing the convergence of one of our NLTE TLUSTY model, and for all the discussions with him. G.P. is also thankful to Simon Jeffery for making available Armagh LTE and other related codes. Travel support for G.P. to visit UT Austin and McDonald Observatory where a part of this work was carried out was provided by the Isabel McCutcheon Harte Centennial Chair. D.L.L. thanks the Robert A. Welch Foundation for support through grant F-634.

\section{REFERENCES}

Abia, C., Pavlenko, Y., \& de Laverny, P. 1999, A\&A, 351, 273

Asplund, M., Gustafsson, B., Lambert, D. L., \& Rao, N. K. 2000, A\&A, 353, 287

Auer, L. H., \& Mihalas, D. 1973, ApJ, 184, 151

Blöcker, T. 2001, Ap\&SS, 275, 1

Cameron, A. G. W., \& Fowler, W. A. 1971, ApJ, 164, 111

Clayton, G. C., Geballe, T. R., Herwig, F., Fryer, C., \& Asplund, M. 2007, ApJ, 662,1220

Clayton, G. C., Herwig, F., Geballe, T. R., Asplund, M., Tenenbaum, E. D., Engelbracht, C. W., \& Gordon, K. D. 2005, ApJ, 623, L141

Cunha, K., Hubeny, I., \& Lanz, T. 2006, ApJ, 647, L143

Drilling, J. S., Jeffery, C. S., \& Heber, U. 1998, A\&A, 329, 1019

García-Hernández, D. A., Hinkle, K. H., Lambert, D. L., \& Eriksson, K. 2009, ApJ, 696, 1733

García-Hernández, D. A., Lambert, D. L., Rao, N. K., Hinkle, K. H., \& Eriksson, K. 2010, ApJ, 714, 144

Harrison, P. M., \& Jeffery, C. S. 1997, A\&A, 323, 177

Herwig, F. 2000, A\&A, 360, 952

Herwig, F. 2001, Ap\&SS, 275, 15

Herwig, F. 2006, Proc. Int. Symp. on Nuclear Astrophysics-Nuclei in the Cosmos IX (2006 June 25-30; Geneva: CERN), 206

Hubeny, I. 1988, Comput. Phys. Commun., 52, 103

Hubeny, I., Hummer, D. G., \& Lanz, T. 1994, A\&A, 282, 151

Hubeny, I., \& Lanz, T. 1995, ApJ, 439, 875

Hubeny, I., Lanz, T., \& Jeffery, C. S. 1994, Newsletter on Analysis of Astronomical Spectra, Vol. 20 (TLUSTY and SYNSPEC: a User's Guide; St. Andrews: St. Andrews Univ.), 30

Iben, I., Jr. 1967, ApJ, 147, 624

Iben, I., Kaler Jr, J. B., Truran, J. W., \& Renzini, A. 1983, ApJ, 264, 605

Iben, I., Jr., \& Tutukov, A. V. 1984, ApJS, 54, 335

Iben, I., Tutukov Jr, A. V., \& Yungelson, L. R. 1996, ApJ, 456, 750

Iben, I. Jr, Tutukov, A. V., \& Yungelson, L. R. 1997, ApJ, 475, 291

Jeffery, C. S. 1996, in ASP Conf. Ser. 96, Hydrogen-deficient Stars, ed. C. S. Jeffery \& U. Heber (San Francisco, CA: ASP), 152

Jeffery, C. S. 1998, MNRAS, 294, 391

Jeffery, C. S. 2008, ASP Conf. Ser. 391, Hydrogen-deficient Stars, ed. K. Werner \& T. Rauch (San Francisco, CA: ASP), 53

Jeffery, C. S., Hamill, P. J., Harrison, P. M., \& Jeffers, S. V. 1998, A\&A, 340, 476

Jeffery, C. S., \& Harrison, P. M. 1997, A\&A, 323, 393

Jeffery, C. S., \& Heber, U. 1992, A\&A, 260, 133

Jeffery, C. S., \& Heber, U. 1993, A\&A, 270, 167

Jeffery, C. S., Hill, P. W., \& Heber, U. 1999, A\&A, 346, 491

Jeffery, C. S., Woolf, V. M., \& Pollacco, D. L. 2001, A\&A, 376, 497

Karakas, A. I. 2010, MNRAS, 403, 1413

Karakas, A. I., Campbell, S. W., \& Stancliffe, R. J. 2010, ApJ, 713, 374

Lambert, D. L., \& Rao, N. K. 1994, JA\&A, 14, 47

Lanz, T., \& Hubeny, I. 2003, ApJS, 146, 417

Lanz, T., \& Hubeny, I. 2007, ApJS, 169, 83

McCarthy, J. K., Sandiford, B. A., Boyd, D., \& Booth, J. 1993, PASP, 310, 881

Morel, T., \& Butler, K. 2008, A\&A, 487, 307

Nieva, M. F., \& Przybilla, N. 2008, A\&A, 481, 199

Pandey, G. 2006, ApJ, 648, L143

Pandey, G., Lambert, D. L., Jeffery, C. S., \& Rao, N. K. 2006, ApJ, 638, 454

Pandey, G., Lambert, D. L., \& Rao, N. K. 2008, ApJ, 674, 1068

Pandey, G., Rao, N. K., Lambert, D. L., Jeffery, C. S., \& Asplund, M. 2001, MNRAS, 324, 937

Pandey, G., \& Reddy, B. E. 2006, MNRAS, 369, 1677

Popper, D. M. 1942, PASP, 54, 160

Rao, N. K., \& Lambert, D. L. 1996, ASP Conf. Ser. 96, Hydrogen-deficient Stars, ed. C. S. Jeffery \& U. Heber (San Francisco, CA: ASP), 43 
Rao, N. K., \& Lambert, D. L. 2008, MNRAS, 384, 477

Rao, N. K., Sriram, S., Jayakumar, K., \& Gabriel, F. 2005, JA\&A, 26, 331

Rao, N. K., et al. 2004, Asian J. Phys., 13, 267

Ryde, N., \& Lambert, D. L. 2004, A\&A, 415, 559

Saio, H., \& Jeffery, C. S. 2002, MNRAS, 333, 121

Schönberner, D. 1979, A\&A, 79, 108

Seaton, M. J. 1998, J. Phys. B: At. Mol. Opt. Phys, 31, 5315

Thackeray, A. D., \& Wesselink, A. J. 1952, Observatory, 72, 248
Tull, R. G., MacQueen, P. J., Sneden, C., \& Lambert, D. L. 1995, PASP, 107, 251

Webbink, R. F. 1984, ApJ, 277, 355

Werner, K., \& Herwig, F. 2006, PASP, 118, 183

Werner, K., Rauch, T., Reiff, E., \& Kruk, J. W. 2008, ASP Conf. Ser. 391, Hydrogen-deficient Stars, ed. K. Werner \& T. Rauch (San Francisco, CA: ASP), 109

Wiese, W. L., Smith, M. W., \& Glennon, B. M. 1966, NSRDS-NBS 4 (Washington, DC: National Buereau of Standards) 